\title{
Engineering cryogenic setups for 100-qubit scale superconducting circuit systems
}

\author{
S. Krinner ${ }^{1 *}$ D, S. Storz ${ }^{1}$, P. Kurpiers ${ }^{1}$, P. Magnard ${ }^{1}$, J. Heinsoo ${ }^{1}$, R. Keller ${ }^{1}$, J. Lütolf ${ }^{1}$, C. Eichler ${ }^{1}$ and A. Wallraff ${ }^{1}$
}

${ }^{\text {*Correspondence: }}$ skrinner@phys.ethz.ch

${ }^{1}$ Department of Physics, ETH Zürich, Zürich, Switzerland

\begin{abstract}
A robust cryogenic infrastructure in form of a wired, thermally optimized dilution refrigerator is essential for solid-state based quantum processors. Here, we engineer a cryogenic setup, which minimizes passive and active heat loads, while guaranteeing rapid qubit control and readout. We review design criteria for qubit drive lines, flux lines, and output lines used in typical experiments with superconducting circuits and describe each type of line in detail. The passive heat load of stainless steel and NbTi coaxial cables and the active load due to signal dissipation are measured, validating our robust and extensible concept for thermal anchoring of attenuators, cables, and other microwave components. Our results are important for managing the heat budget of future large-scale quantum computers based on superconducting circuits.
\end{abstract}

\section{Introduction}

Promising solid-state based quantum computing platforms, such as superconducting circuits [1] or charges and spins in semiconductor quantum dots [2], require temperatures on the Millikelvin level to initialise the systems in their ground state and to avoid errors due to thermal excitation during operation. Millikelvin temperatures are achieved in $\mathrm{He}^{3} / \mathrm{He}^{4}$ dilution refrigerators (DR). When scaling those approaches from the few qubit level to large scale quantum processors, an increasing number of microwave and DC cables need to be integrated into the dilution refrigerator. They connect the classical control electronics at room temperature (RT) to the quantum processor at the lowest temperature stage of the dilution refrigerator, creating a substantial heat load on the dilution refrigerator due to heat conduction. Besides this passive load, active load due to the dissipation of control signals in cables and attenuators plays a major role. Engineered dissipation is necessary to thermalize the incoming radiation fields and to reduce the number of thermal photons incident on the sample $[3,4]$.

While superconducting quantum processors operating 20 qubits have been realized [ 5 , 6], their cryogenic setup and extensibility towards larger system sizes has not been reported on. Here, we present a thermally optimized, robust cabling scheme and cryogenic setup suitable for the operation of 50 qubits at a temperature of $14 \mathrm{mK}$. Disregarding space constraints in the dilution refrigerator, at least 150 qubits could be operated using our approach. Before describing our customized cryogenic system in Sect. 3, we put forward methods for minimizing passive heat load on all stages of the dilution refrigerator and for minimizing the number of thermal photons in cables connecting room temperature

(c) The Author(s) 2019. This article is distributed under the terms of the Creative Commons Attribution 4.0 International License (http://creativecommons.org/licenses/by/4.0/), which permits unrestricted use, distribution, and reproduction in any medium, provided you give appropriate credit to the original author(s) and the source, provide a link to the Creative Commons license, and indicate if changes were made. 
components of the setup to the base temperature stage while keeping the active load low (Sect. 2). We then present measurements of the passive load arising from the installed cable trees comprising typically 25 coaxial lines each, and compare them to estimates based on available data on thermal conductances of coaxial cables (Sect. 4). Active load due to application of control signals is characterized in Sect. 5. Finally, we discuss the total heat load in Sect. 6 along with possible improvements which we expect to allow to increase the number of operational qubits to up to one thousand when disregarding space constraints.

\subsection{Typical cabling for experiments with superconducting circuits}

When designing the control lines and output lines connecting to a superconducting quantum processor the goal is to provide sufficiently strong coupling rates to the quantum processor, while minimizing decoherence due to coupling of the quantum processor via these lines to its environment. Thermal noise, present due to the connection of the quantum processor to electronics at room temperature, not only leads to qubit dephasing $[3,4,7]$, but can also lead to creation of quasi-particles and thus to dissipation and reduced energy relaxation times [8-13]. Hence, thorough thermalization of cables, attenuators, and microwave components at the various temperature stages of the dilution refrigerator is not only important for reducing the heat load on the dilution refrigerator, but also for protecting the quantum processor from thermal radiation. In addition to thermal anchoring, filters with stop-bands outside the frequency range of qubits and readout resonators as well as infra-red blocking filters $[12,14,15]$ further suppress thermal radiation. Other sources of external noise that can negatively impact coherence times include $1 / \mathrm{f}$ noise [ 7 , 16] from electronic instrumentation and magnetic field fluctuations or magnetic offset fields inducing magnetic vortices that can lead to dissipation [12, 17, 18]. These sources can be mitigated with appropriate filters and magnetic shielding respectively, but their study is not at the focus of the present work.

We briefly present an overview of the cabling typically used in experiments with superconducting circuits. We distinguish between direct-current (DC) and radio-frequency (RF) cabling. DC lines are made from twisted pairs of wires, that are low-pass filtered, and thermalized at each temperature stage. Typical uses are biasing of cryogenic amplifiers and flux biasing of frequency-tunable qubits [19]. RF lines on the other hand are realized as semi-rigid microwave cables and contain various microwave components such as attenuators, filters and amplifiers. They connect to the quantum processor and are used for its control and readout. One typically distinguishes between drive lines, flux lines, and output lines, which we briefly describe here.

Drive lines are used for controlling the quantum states of qubits with a microwave tone realizing single-qubit gates, and for probing the frequency shift of readout resonators. To reduce thermal population of qubits, and frequency shifts of the qubits due to their dispersive interaction with a readout resonator [20], the number of thermal noise photons in the drive lines arriving at the mixing chamber plate $(\mathrm{MXC})$ of the dilution refrigerator is required to be well below the single photon level in both cases. More precisely, to guarantee a noise photon number at MXC on the $10^{-3}$ level, a total attenuation of about $60 \mathrm{~dB}$ is required, see Sect. 2.2.1 and 3.2. At the same time, the bandwidth of the drive lines is required to be large enough to cover the typical frequency ranges of qubits $(4-6 \mathrm{GHz})$ and of readout resonators $(4-8 \mathrm{GHz})$.

Flux lines are used for implementing two-qubit gates which are based on the dynamical flux tunability of the transition frequency of a qubit [21-23] or of a separate coupling 
subcircuit $[24,25]$. In addition, qubit frequency variations, occurring due to imperfections in the fabrication of Josephson junctions, can be compensated. Tunable frequency qubits, as opposed to fixed frequency qubits, make use of a SQUID (superconducting quantum interference device) loop instead of a single Josephson junction as the inductive element. By threading a magnetic flux $\Phi$ through this loop the frequency of the qubit approximately changes as $\omega_{\mathrm{q}} \simeq \omega_{0} \sqrt{\left|\cos \left(\pi \Phi / \Phi_{0}\right)\right|}[16]$, where $\Phi_{0}$ is the magnetic flux quantum. A mutual inductance between flux line and SQUID loop is realized by routing the on-chip part of the flux line past the SQUID loop. Hence, a current applied to the flux line results in a magnetic flux in the SQUID loop, effectively tuning the transition frequency of the qubit. Low-pass filters in the flux lines limit the bandwidth to about $1 \mathrm{GHz}$ eliminating thermal noise at qubit frequencies. However, since the magnetic flux $\Phi$ in the SQUID loop sets the qubit transition frequency, any current noise leads to qubit dephasing $[7,16]$. To reduce the current noise, a suitable amount of attenuation $(10-20 \mathrm{~dB})$ is also added in the flux lines, see Sect. 3.3.

Output lines contain a series of cryogenic and room temperature amplifiers for the detection of readout signals [26-28]. To isolate the sample from thermal noise photons and from input noise of amplifiers while not attenuating the output signal, isolators and circulators are used, see Sect. 3.4 for more details.

\section{Sources of heat loads}

We consider three dominant contributions to the heat load on the dilution refrigerator. First, passive load is due to heat flow from higher temperature stages to lower temperature stages. Here, we consider only heat conducted through installed cables. Heat which flows via posts that separate the various plates of the dilution refrigerator is not considered because it is already taken into account in the available cooling power of the dilution refrigerator specified by the manufacturer and separately evaluated in this work [29-31]. Second, active load arises due to the dissipation (Joule heating) of applied microwave signals in attenuators and in the microwave cables themselves. Dissipation arising from DC signals, used e.g. to bias HEMT amplifiers at the $4 \mathrm{~K}$ stage or to flux bias qubits on the chip at MXC, falls also in this category. Third, a radiative load arises due to blackbody radiation from stages and shields of higher temperature impinging on stages and shields of lower temperature [32]. This load, however, is also taken into account in the specified available cooling power of the dilution refrigerator. For completeness, we mention load due to residual gas, in particular Helium since it has the highest vapor pressure at cryogenic temperatures. However, during normal operation of the dilution refrigerator, the cryo-pumping capacity of the cold surfaces in the dilution refrigerator keeps the pressure in the vacuum can below $10^{-5}$ mbar providing an adequate isolation vacuum [32]. We therefore do not consider this load. However, latent heat released during desublimation processes can create additional loads, e.g. when gas desorbs from installed components and freezes out at the closest stage.

\subsection{Passive load}

To minimize passive heat load we use cable materials with low thermal conductivity. We note that with the exception of superconductors this typically goes along with poor electrical conductivity, and hence leads to more dissipation when microwave signals are applied. However, in most of the lines attenuation and thus dissipation is desired anyway to thermalize the incoming radiation fields, see Sect. 2.2. 
To estimate the passive load due to installed cables we consider fixed temperatures on the plates of the dilution refrigerator, corresponding to a steady state, in which the heat flows onto the various stages are absorbed by the cooling powers available at those stages. Note that the cooling power on a given stage depends on the temperature of that stage. Integrating Fourier's law [33] yields the heat flow $P_{i}$ to stage $i$ with temperature $T_{i}$ due to a single coaxial cable,

$$
P_{i}=\int_{T_{i-1}}^{T_{i}} d T \frac{\rho_{\mathrm{o}}(T) A_{\mathrm{o}}+\rho_{\mathrm{d}}(T) A_{\mathrm{d}}+\rho_{\mathrm{c}}(T) A_{\mathrm{c}}}{L_{i}}
$$

where $\rho_{\mathrm{o}}, \rho_{\mathrm{d}}, \rho_{\mathrm{c}}$, and $A_{\mathrm{o}}, A_{\mathrm{d}}, A_{\mathrm{c}}$ are thermal conductivities and cross sections of outer conductor, dielectric, and center conductor respectively, and $L_{i}$ is the length of the cable connecting stage $i-1$ and $i . P_{i}$ has units of power. The labeling $i \in\{1,2,3,4,5\}$ corresponds to the stages \{“50 K", " $4 \mathrm{~K}$ ", "Still”, Cold plate (“CP”), "MXC"\}. Table 1 lists the corresponding temperatures in our Bluefors XLD400 dilution refrigerator as achieved in the empty dilution refrigerator after installation. The values are typical for state of the art pulse tube cooler based dilution refrigerators [34,35]. The thermal conductivities used for the estimates in this paper are listed in Appendix 1.

To motivate the choice of cable materials detailed in Sect. 3 we show the heat flow to the various temperature stages due to single coaxial cables of different material in Fig. 1. We note that these estimates assume thermalization of outer conductor, dielectric, and center conductor at every stage, see Sect. 4.2 for a discussion.

Table 1 Dilution refrigerator specifications. Temperatures and available cooling powers on the indicated stages of a Bluefors XLD400 DR. Coaxial cable lengths towards the respective stages are listed as well

\begin{tabular}{llll}
\hline Stage name & Temperature $(\mathrm{K})$ & Cooling power $(\mathrm{W})$ & Cable length $(\mathrm{mm})$ \\
\hline $50 \mathrm{~K}$ & 35 & $30($ at $45 \mathrm{~K})$ & 200 \\
$4 \mathrm{~K}$ & 2.85 & $1.5($ at $4.2 \mathrm{~K})$ & 290 \\
Still & $882 \times 10^{-3}$ & $40 \times 10^{-3}$ (at $\left.1.2 \mathrm{~K}\right)$ & 250 \\
CP & $82 \times 10^{-3}$ & $200 \times 10^{-6}($ at $140 \mathrm{mK})$ & 170 \\
MXC & $6 \times 10^{-3}$ & $19 \times 10^{-6}($ at $20 \mathrm{mK})$ & 140 \\
\hline
\end{tabular}

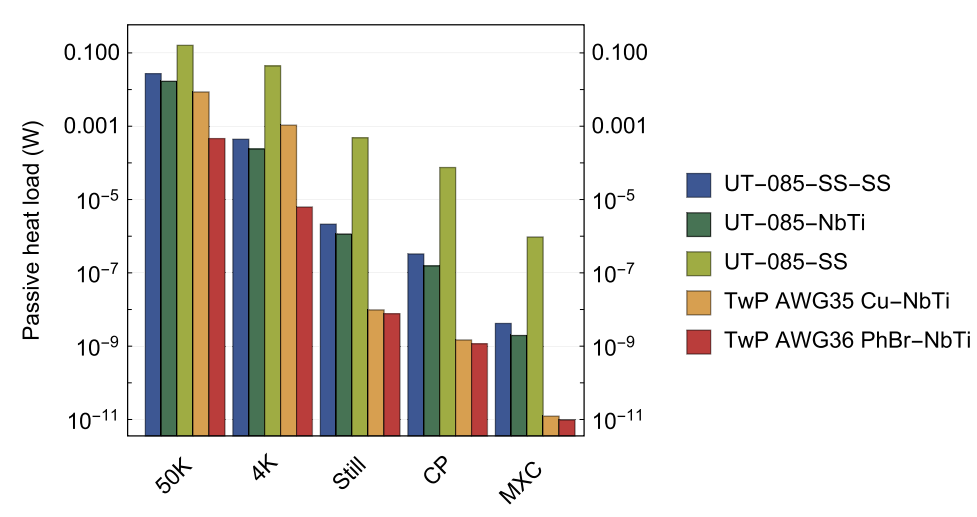

Figure 1 Heat flow for indicated cable types. Heat flow to the five temperature stages for indicated cable types and given cable lengths (see text). Twisted pairs (TwP) consist of either $\mathrm{Cu}(\mathrm{RRR}=100$ ) or PhBr from RT to the $4 \mathrm{~K}$ stage, and of NbTi from the $4 \mathrm{~K}$ to the MXC stage 
Three typical and readily available types of $0.085^{\prime \prime}$ diameter coaxial cable are discussed. The lengths of the cables are listed in Table 1 and correspond to the inter-plate distances in the dilution refrigerator plus a small correction due to the bends used for stress and strain relief in those cables (see Sect. 3). Stainless steel cables (UT-085-SS-SS) and niobiumtitanium cables (UT-085-NbTi) have the lowest passive load. Therefore, we choose stainless steel cables for the drive lines where large attenuation is needed. NbTi cable is superconducting below $10 \mathrm{~K}$ and therefore has very low attenuation below $10 \mathrm{~K}$ [36], which is why we use it for the output lines in the sections between the $4 \mathrm{~K}$ and MXC stages. The heat flows associated with these two cable types are dominated by their outer conductor, which has a cross-sectional area that is by a factor 10 larger than the cross-sectional area of the center conductor. The contribution of the Teflon dielectric is of the same order of magnitude as the one of the inner conductor, which is due to the low thermal conductivity of Teflon, see Appendix 1, and the cross-sectional area of the dielectric being comparable to the one of the outer conductor.

An alternative to stainless steel is cupronickel $(\mathrm{CuNi})$ cable. It is expected to have a passive load which is about $50 \%$ larger than the one of SS-SS cable [37]. Another commonly used cable type is UT-085-SS cable (stainless steel outer conductor, SPCW ${ }^{\mathrm{a}}$ center conductor), which results in a significantly larger heat load due to the larger thermal conductivity of $\mathrm{Cu}$, which is anomalously enhanced at temperatures below $50 \mathrm{~K}$, see Appendix 1 . Due to its lower attenuation, see Appendix 2, this cable type is in principle suited for output lines in the sections from the vacuum flange of the dilution refrigerator to the $4 \mathrm{~K}$ stage, see discussion in Sect. 3.4.

In our dilution refrigerator the $\mathrm{DC}$ wiring was pre-installed by the manufacturer. Copper or phosphor-bronze (PhBr) twisted pairs of diameters AWG35 and AWG36 respectively are only used from room temperature to the $4 \mathrm{~K}$ stage due to their large thermal conductivity. From $4 \mathrm{~K}$ to MXC superconducting NbTi twisted pairs are used. ${ }^{\mathrm{b}}$ Among these materials only $\mathrm{Cu}$ twisted pairs cause a significant passive load comparable to the one of the coaxial cables, see Fig. 1.

\subsection{Active load}

The active load in the dilution refrigerator depends on the level of attenuation of the RF lines and the installed attenuators, and on the signal levels required at the chip. Therefore, in this section, we briefly discuss how much attenuation is needed to reduce thermal noise and how to distribute the attenuators among the various temperature stages. Furthermore, we discuss the signal levels required in typical experiments with superconducting qubits.

\subsubsection{The need of attenuation}

Although the signals required to drive qubits or to read them out are small (e.g. a peak power of $-66 \mathrm{dBm}$ for a $20 \mathrm{~ns}$ long $\pi$-pulse), the power applied at the input of the dilution refrigerator is orders of magnitude larger. This is because a total attenuation of at least $\sim 60 \mathrm{~dB}$ is required to reduce blackbody radiation present in cables at room temperature to a level corresponding to a thermal photon occupation number of a few $10^{-3}$ at the sample. The blackbody radiation in effectively one-dimensional cables is known as Johnson-Nyquist noise in electronics. If a resistor $R$ is connected to the cable or if the cable is coaxial with a characteristic impedance $R$ the induced voltage fluctuations $\delta V(t)$ 
have a power spectral density given by [38]

$$
S_{V}^{\mathrm{th}}(T, \omega)=\int_{-\infty}^{\infty} \mathrm{d} t\langle\delta V(0) \delta V(t)\rangle \mathrm{e}^{-i \omega t}=2 R \hbar \omega \frac{1}{\exp \left(\hbar \omega / \mathrm{k}_{\mathrm{B}} T\right)-1} .
$$

The last factor in this equation is the photon occupation number as dictated by the Bose-Einstein distribution $n_{\mathrm{BE}}(T, \omega)=1 /\left[\exp \left(\hbar \omega / \mathrm{k}_{\mathrm{B}} T\right)-1\right]$. It is dimensionless and can be thought of as photon flux spectral density, i.e. as number of photons per $\mathrm{Hz}$ frequency interval, per second. In the classical limit $\hbar \omega \ll \mathrm{k}_{\mathrm{B}} T$ one recovers the original (two-sided) Johnson Nyquist formula $S_{V}^{\text {th }}(T)=2 \mathrm{k}_{\mathrm{B}} T R$.

In a coaxial cable connecting room temperature electronics to base temperature circuits, thermal photons propagate down the line towards the lower temperature stages. To reduce the spectral density of thermal radiation a series of attenuators is installed in the microwave line. An attenuator with an attenuation of $A=20 \mathrm{~dB}=100$ effectively acts as a beamsplitter which transmits $1 \%$ of the incident signal and adds $99 \%$ of blackbody radiation with the effective temperature $T_{\text {att }}$ at which the attenuator is thermalized. We note that $99 \%$ of the incident signal is dissipated in the attenuator. To prevent the attenuator from heating up and to keep the effective temperature at which it re-emits thermal radiation low it is efficiently thermalized. More formally, the noise photon occupation number $n_{i}$ at stage $i$ with attenuation $A_{i}$ is given by

$$
n_{i}(\omega)=\frac{n_{i-1}(\omega)}{A_{i}}+\frac{A_{i}-1}{A_{i}} n_{\mathrm{BE}}\left(T_{i, \mathrm{att}}, \omega\right) .
$$

When installing a cascade of attenuators in the microwave line thermalized at subsequently lower temperature stages, we employ this relation to determine the noise photon occupation number $n_{\mathrm{MXC}}$ at the MXC stage.

For the discussion presented here we consider the signal frequency at $\bar{\omega} / 2 \pi=6 \mathrm{GHz}$ since typical qubits are operated at $5 \mathrm{GHz}$ and typical readout resonators at $7 \mathrm{GHz}$. A lower bound for the total attenuation needed to achieve a noise photon number of $n_{\mathrm{MXC}}=10^{-3}$ is obtained by neglecting the second term on the r.h.s. of Eq. (3) yielding $n_{\mathrm{BE}}(T=300 \mathrm{~K}, \bar{\omega}) / 10^{-3}=60 \mathrm{~dB}$. This is a lower bound since blackbody radiation emitted by attenuators at all other temperature stages is neglected.

Distributing the total attenuation among the various temperature stages is important to keep the active load on the lower stages well below the cooling powers available at those stages. In distributing the attenuation we avoid putting more attenuation $A_{i}$ on stage $i$ than is needed to thermalize the incoming radiation fields onto that stage, i.e. the first term on the r.h.s. of Eq. (3) should not be significantly smaller than the value to which the second term saturates for $A_{i} \gg 1$. Hence, good reference values for the attenuation on stage $i$ are $A_{i \text {,ref }}=n_{\mathrm{BE}}\left(T_{i-1}, \bar{\omega}\right) / n_{\mathrm{BE}}\left(T_{i}, \bar{\omega}\right)$. As an example we consider a total attenuation of $60 \mathrm{~dB}$ that is composed of $20 \mathrm{~dB}$ attenuators thermalized at the $4 \mathrm{~K}, \mathrm{CP}$, and MXC stages. To gain some further insight, we first plot $n_{\mathrm{MXC}}$ as a function of $A_{4 \mathrm{~K}}$ for a fixed attenuation of $20 \mathrm{~dB}$ at the $\mathrm{CP}$ and MXC stages respectively, see blue solid line in Fig. 2. We observe that the number of noise photons is efficiently reduced by increasing the attenuation up to $\sim 20 \mathrm{~dB}$, as expected from the reference value $A_{4 \mathrm{~K} \text {, guess }}=n_{\mathrm{BE}}\left(T_{\mathrm{RT}}, \bar{\omega}\right) / n_{\mathrm{BE}}\left(T_{4 \mathrm{~K}}, \bar{\omega}\right) \approx$ $T_{\mathrm{RT}} / T_{4 \mathrm{~K}}=300 / 3=20 \mathrm{~dB}$. More attenuation is unnecessary because at this value the radiation field incident from room temperature has reached the $4 \mathrm{~K}$ thermal noise floor. 

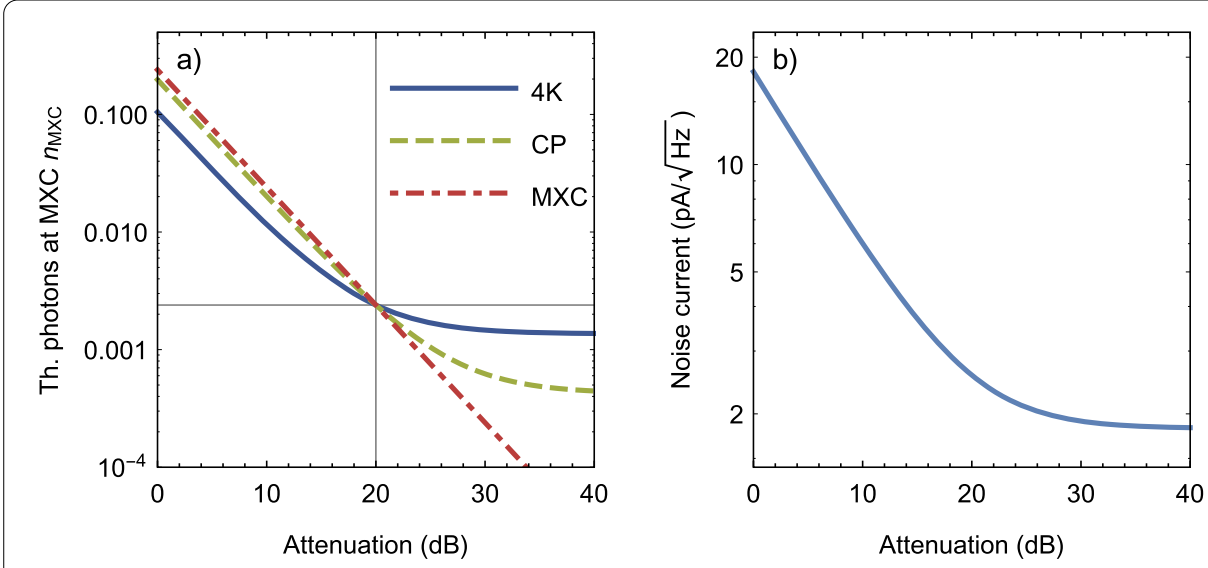

Figure 2 Thermal noise photon numbers. (a) Calculated thermal photon number at MXC as a function of the degree of attenuation at the $4 \mathrm{~K}$ stage (blue), CP (green), MXC (red) for a fixed attenuation of $20 \mathrm{~dB}$ at the respective other stages ( $45 \mathrm{~K}$ stage and $1 \mathrm{~K}$ stage have no attenuators in this example). The calculations are based on Eq. (3). (b) Thermal noise current spectral density at MXC as a function of attenuation at the $4 \mathrm{~K}$ stage, calculated using Eq. (4)

We observe a similar feature when plotting $n_{\mathrm{MXC}}$ as a function of $A_{\mathrm{CP}}$, for a fixed attenuation of $20 \mathrm{~dB}$ at the $4 \mathrm{~K}$ and MXC stages respectively (green dashed line in Fig. 2). Here a little bit more than $20 \mathrm{~dB}$ attenuation would further reduce $n_{\mathrm{MXC}}$, as expected from a reference value $A_{\mathrm{CP} \text {,ref }}=n_{\mathrm{BE}}\left(T_{4 \mathrm{~K}}, \bar{\omega}\right) / n_{\mathrm{BE}}\left(T_{\mathrm{CP}}, \bar{\omega}\right)=22 \mathrm{~dB}$. We finally plot $n_{\mathrm{MXC}}$ as a function of attenuation at MXC (red dash-dotted line in Fig. 2). It demonstrates that, over the considered attenuation range, adding more attenuation at MXC linearly decreases $n_{\mathrm{MXC}}$. The reason is that the noise floor of thermal photons at MXC is as low as $n_{\mathrm{BE}}\left(T_{\mathrm{MXC}}=20 \mathrm{mK}, \bar{\omega}\right)=6 \times 10^{-7}$. However, we will see in Sect. 3.2 that the limited cooling power at MXC prevents us from installing significantly more than $20 \mathrm{~dB}$ of attenuation at MXC.

\subsubsection{Signal levels required for the operation of the quantum processor}

In this section we estimate the powers required at the chip for driving a $\pi$-pulse on a qubit and setting a flux bias on a qubit, two important operations on superconducting qubits.

To drive a $\pi$-pulse on a qubit, we apply an RF pulse at the qubit frequency with a Gaussian envelope through a CPW transmission line weakly capacitively coupled to the qubit. This coupling has to be sufficiently small to prevent Purcell decay of the qubit into the transmission line [39]. The finite value of the coupling imposes a limit on the $T_{1}$ time of the qubit, which we target here to be no lower than $T_{1}^{\text {lim }}=2 \mathrm{~ms}$, corresponding to a reduction of the $T_{1}$ time of a qubit with an intrinsic life time of $100 \mu \mathrm{s}$ [5] by no more than $5 \%$. The amplitude of the time-dependent Rabi frequency $\Omega_{R}(t)=\Omega_{0} \exp \left[-t^{2} /\left(2 \sigma^{2}\right)\right]$ for a $20 \mathrm{~ns}$ long $\pi$-pulse [40] with a width $\sigma=3.3 \mathrm{~ns}$ is given by $\Omega_{0}=\sqrt{\pi /\left(2 \sigma^{2}\right)} \approx 2 \pi \times 60 \mathrm{MHz}$. To achieve this value a peak power $P_{\mathrm{p}}=\hbar \omega_{q} T_{1}^{\lim } \Omega_{0}^{2} / 4 \approx-66 \mathrm{dBm}$ is needed [39, 41]. For an estimate of the associated heat load we employ the average power of the pulse, $P_{\text {avg }}=P_{\mathrm{p}} \frac{1}{6 \sigma} \int_{-3 \sigma}^{3 \sigma} \exp \left[-t^{2} / \sigma^{2}\right] \mathrm{d} t=\frac{\sqrt{\pi}}{6} P_{\mathrm{p}} \approx-71 \mathrm{dBm}$. This number will be further reduced due to a finite duty cycle of those pulses during the execution of a quantum algorithm. We assume a maximum duty cycle of $33 \%$, corresponding to an operation mode of the quantum processor where single- and two-qubit gates are alternated with the two-qubit gate duration being twice as long as the single-qubit gate duration [22, 42]. Also $\pi / 2$-pulses 
require only a quarter of the power. Assuming an equal share between $\pi$ - and $\pi / 2$-pulses, we use an average required power per qubit drive line of $-78 \mathrm{dBm}$ for the estimates presented in Sect. 3.2. We note that the duty cycle of drive pulses can be significantly lower than $33 \%$ if the durations of two-qubit gates and readout pulses are significantly longer than the duration of drive pulses. A further reduction of the duty cycle arises if the repetition period of the algorithm is dominated by a wait time to reset the qubits to the ground state.

Readout signals used to drive readout resonators to infer the state of qubits are typically an order of magnitude smaller and have a lower duty cycle. They are therefore not taken into account in our estimates.

Concerning dissipation in flux lines, we primarily consider DC biasing currents, which are constantly applied to set the qubit frequency, in most of the cases to the so-called sweet spot $\omega_{\mathrm{q}}=\omega_{0}$, at which the qubit frequency $\omega_{q}$ is to first-order insensitive to flux noise [16]. Assuming a worst case scenario of random offset magnetic fields in the SQUID loops, the compensating flux $\Phi_{\text {offset }}$ which we apply to reach the closest sweet spot, is equally distributed in the interval $\left[-\Phi_{0} / 2, \Phi_{0} / 2\right]$. This corresponds to a current interval of $[-1,1] \mathrm{mA}$, when using a reasonable mutual inductance of $M=\partial \Phi / \partial I=0.5 \Phi_{0} / \mathrm{mA}$ between the flux line and the SQUID loop. The mutual inductance is determined by the coupling strength (proximity) of the on-chip flux line to the SQUID loop and the area of the SQUID loop. A large $M$ on the one hand minimizes the required current and thus dissipation in the line, and allows for the use of low-noise current sources which typically have a small dynamic range. On the other hand, a large SQUID loop is also more susceptible to (external) magnetic flux noise, and care needs to be taken in the design of large coupling strengths in order to keep residual capacitive coupling of the qubit to the flux line low, which otherwise can limit the qubit lifetime due to the Purcell effect [16]. The dissipated heat due to the bias currents is most critical at the MXC stage which has the lowest cooling power. The amount of dissipation depends on the DC resistances of the stainless steel cable and low-pass filter installed between $\mathrm{CP}$ and MXC, and on the stage to which the dissipated heat predominantly flows to. We discuss the experimental determination of this heat load in Sect. 5.2.

We also consider dissipation due to flux pulses, which are used for the realization of two-qubit gates. Assuming that during the flux pulse the qubit frequency is tuned by about $10 \%$ of its sweet spot value [21,22], a flux amplitude of $\pm 0.2 \Phi_{0}$ is needed, corresponding to a current amplitude of $0.4 \mathrm{~mA}$. The associated active loads are estimated in Sect. 5.2 using the results from the DC measurements. To estimate the duty cycle of flux pulses we consider alternating single- and two-qubit gates, as discussed above, and flux pulses applied only to one of the qubits during a two-qubit gate, yielding a duty cycle of $0.5 \times$ $66 \%=33 \%$.

\subsection{Radiative load}

Each temperature stage $i$ except for the cold plate is fitted with a dedicated heat shield to protect the next lower temperature stage $i+1$ from radiative load from temperature stage $i-1$. For a given temperature stage $i$ we calculate the radiative heat load on heat shield $i$ from the enclosing heat shield by assuming infinitely extended cylindrical heat shields, and solving a system of coupled heat equations. The heat shields at the $50 \mathrm{~K}$ and $4 \mathrm{~K}$ stages are made of Aluminium, and of $\mathrm{Cu}$ on Still and MXC. They are characterized by their emissivity $\epsilon=0.06$, as quoted by the manufacturer. Compared to the cooling power 
of each stage, we find a significant contribution only for the $50 \mathrm{~K}$ stage, amounting to $\sim 50 \mathrm{~W}$. This corresponds to about half of the nominal cooling power of the two pulse tube coolers (2x Cryomech PT 420). This load however has already been taken into account in the available cooling power as specified by the manufacturer (40-50 W). Radiative loads at the lower temperature stages are insignificant [32].

\section{Cryogenic setup}

\subsection{Dilution refrigerator (DR)}

A customized and now commercially available version of a Bluefors XLD400 dilution refrigerator as described in this paper is shown in Fig. 3(a). It has a dilution unit positioned in the center of the MXC plate, making space available for an additional line-of-sight (LOS) port at the circumference of the system. A total of six LOS side ports are available in the system for the installation of RF cables. A LOS port consists of a series of vertically aligned cutouts in the plates for feeding through RF cables. In each cutout we install Cu plates for the thermalization of cables and attenuators, see Fig. 4(a)-(c). Instead of standard circular cutouts we designed rectangular cutouts with a width of $88 \mathrm{~mm}$. They allow for mounting fully assembled cable trees from the side using a custom mounting tool, see Fig. 4(e)-(g). This has the advantage that each cable tree, comprising of $25 \mathrm{RF}$ lines and the vacuum flange, can be pre-assembled and tested outside of the dilution refrigerator. Two of the eight sectors at the circumference at the $50 \mathrm{~K}$ and $4 \mathrm{~K}$ stages are occupied by two pulse tube coolers. We make use of the corresponding cutouts on the Still, CP, MXC stages below the pulse tube coolers for installing readout components, as detailed below.

Furthermore, the distance between the Still plate and the $\mathrm{CP}$ has been enlarged by $60 \mathrm{~mm}$ to provide more space for the inline integration of MW components, and for re-

a)

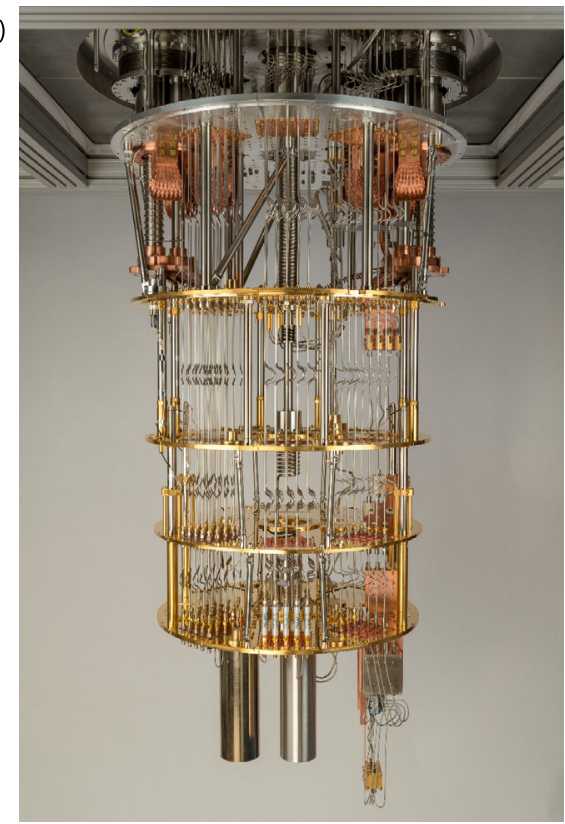

b)

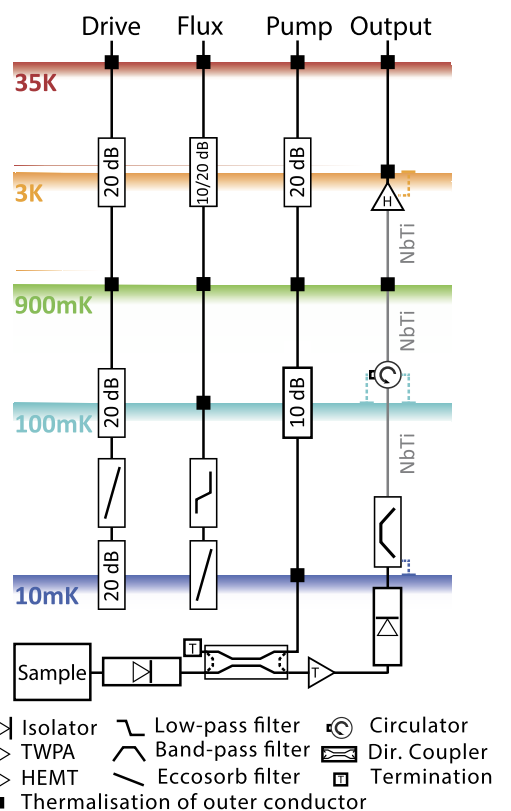

Figure 3 Cabled dilution refrigerator (DR). (a) Bluefors XLD DR with 25 drive lines, 25 flux lines, 4 read-out, 6 read-in, and 5 pump lines installed (see end of Sect. 3.1 for details). (b) Schematic of the cabling configuration within the DR 

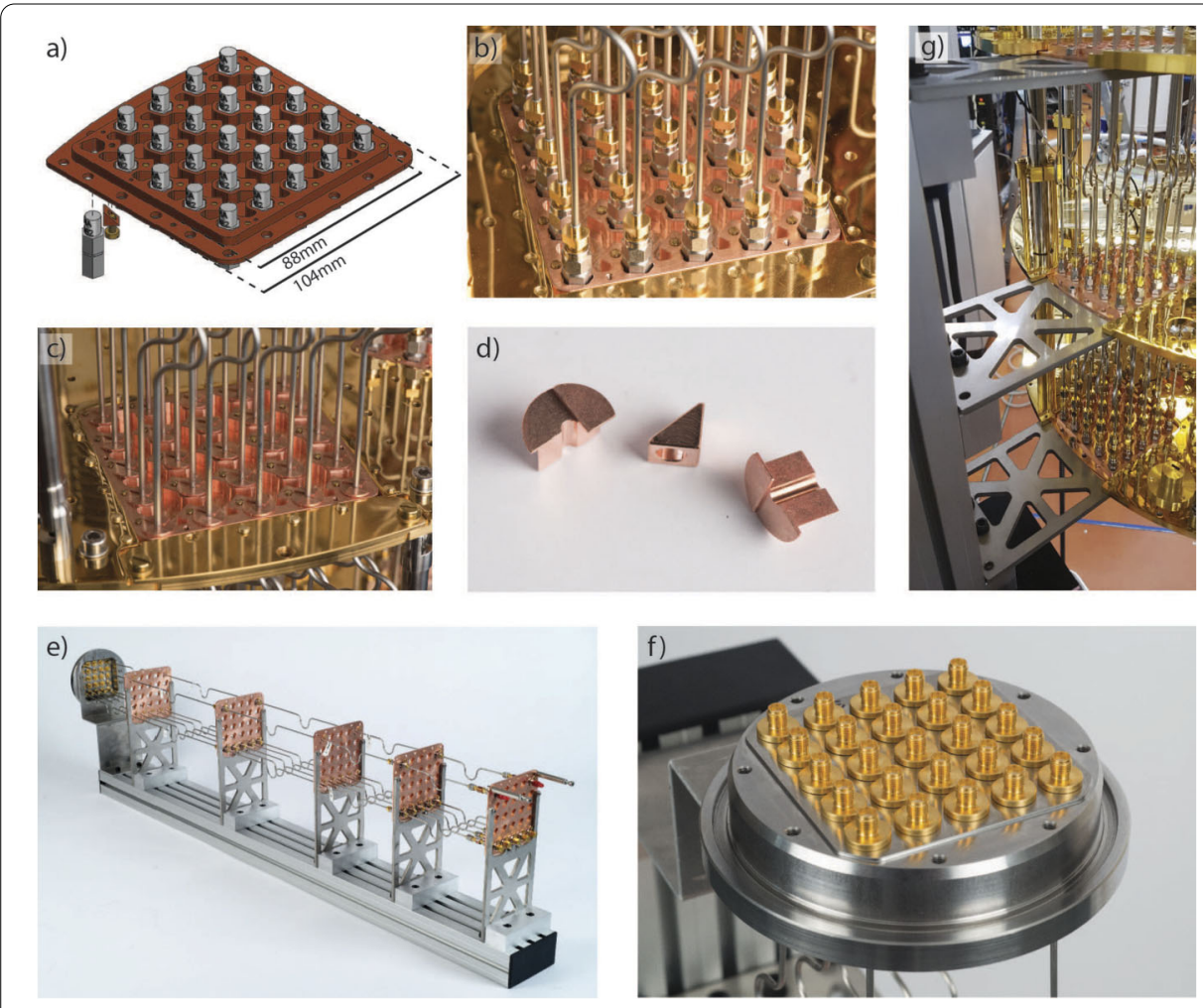

Figure 4 Components for thermalization of RF cables and attenuators, and pre-assembly of cable trees. (a) CAD drawing of the Cu plate used to mount and thermalize attenuators. To clamp the attenuators to the copper plate, an adapter as shown in (d) is used. The Cu plates are then fixed on the corresponding plate of the DR using screws. (b) Photograph of a mounting plate with cabling installed in the DR. (c) Photograph of the same plate, here used to clamp RF cables using the radiation tight Cu adapter pieces shown in (d). (d) Two-part Cu adapter which is pressed around an RF cable using the wedge shaped piece in the middle in combination with a screw (see also (a))). (e) Preassembly tool with mounted Cu plates and vacuum flange. (f) Vacuum flange with vacuum tight SMA adapters installed. (g) Mounting the preassembled cable tree into the DR using a custom mounting tool

ducing the passive heat load due to the cables from Still to CP. The dilution refrigerator was pre-equipped by the manufacturer according to our specifications with two looms of Cu twisted pairs (AWG 35) and two looms of Ph-Br twisted pairs (AWG 36), running both from room temperature to $4 \mathrm{~K}$. From $4 \mathrm{~K}$ to MXC two looms of NbTi twisted pairs were pre-installed. Each of the looms contains 12 twisted pairs.

We have measured the available cooling power on each stage of our custom dilution refrigerator (in the presence of the pre-installed DC wiring), see Table 1, using the technique described in Sect. 4.1. These powers are not to be exceeded to guarantee a base temperature below $20 \mathrm{mK}$ [43]. We designed and planned the wiring and attenuation scheme of the dilution refrigerator with the goal that the resulting heat load is at least about a factor three smaller than those cooling powers. The table also contains the RF cable lengths between the stages. Note that the cables have bends (bending radius $\sim 1 \mathrm{~cm}$ ) to reduce strain on RF connectors when the system is cooled down.

As discussed in Sect. 2, good thermalization of the cables and attenuators is important for both reducing thermal noise photons affecting qubit coherence and reducing the heat load on the lower temperature stages affecting the cryostat performance. For this purpose, we have developed a common thermalization method for both attenuators and cables. It 
consists of a $\mathrm{Cu}$ plate with hexagonal cutouts, in which we individually clamp attenuators using a wedge shaped $\mathrm{Cu}$ piece, which presses the attenuator against two faces of the cutout when tightening a three millimeter (M3) Brass screw (Fig. 4(a) and (b)). We apply a torque of $0.9 \mathrm{Nm}$ on each screw for fastening. We use Brass screws as they thermally contract more than $\mathrm{Cu}$ when cooled down, providing additional clamping force to the attenuator. This is important, because in general the contact resistance between two metal pieces at low temperatures is dominated by clamping force rather than surface area [44]. In addition to thermalizing attenuators we thermalize the outer conductors of RF cables at stages without attenuators using the same $\mathrm{Cu}$ plate (Fig. 4(c)) in combination with a two-part $\mathrm{Cu}$ adapter (Fig. 4(d)). The round flange on those pieces guarantees radiation tight mounting. Using those plates we fit 25 RF lines into each of the six LOS ports.

We now continue to describe in more detail the custom cabling of the dilution refrigerator. Schematics of drive lines, flux lines, and lines for readout are shown in Fig. $3 \mathrm{~b}$ and are typical for superconducting qubit experiments. To minimize passive heat load, we choose UT-085-SS-SS cables for all types of lines except for the output lines. ${ }^{\mathrm{c}}$ We characterize and test the system at half of its capacity by installing three out of six possible cable trees in the dilution refrigerator: a cable tree comprising of 25 drive lines, a cable tree comprising of 25 flux lines, and a cable tree comprising of lines needed for readout. This readout cable tree consists of six drive lines and 5 pump lines (see Sect. 3.4), running both from top to bottom of the dilution refrigerator, and four output lines running from room temperature to $4 \mathrm{~K}$. The remaining parts of the output lines continue in the non-LOS cutouts below one of the pulse tube coolers, where more space is available for larger components of the output lines.

\subsection{Drive line configuration}

Based on the discussion of thermal noise presented in Sect. 2.2.1 here we motivate our choice of attenuator configuration of $20 \mathrm{~dB}$ attenuators at each of the $4 \mathrm{~K}, \mathrm{CP}, \mathrm{MXC}$ stages. We first evaluate the thermal noise photon number $n_{\mathrm{MXC}}$ at MXC in a coaxial cable connecting the room temperature vacuum flange to $\mathrm{MXC}$, for four attenuator configurations each with $60 \mathrm{~dB}$ of total attenuation, see solid bars in Fig. 5(a). The calculations assume the plate temperatures listed in the second column of Table 1 as effective attenuator temperatures. In addition, we take into account the attenuation in the RF cables, which sum up to about $9 \mathrm{~dB}$ when combining room temperature and low temperature data [36], see Appendix 2. Using a continuous version of Eq. (3) and assuming linear temperature gradients in the cables connecting the stages, we obtain $n_{\mathrm{MXC}}$ as indicated by the dashed bars in Fig. 5(a). For each configuration we calculate the power dissipated in 25 drive lines on each temperature stage and compare the result to the available cooling power on that stage (Table 1), see Fig. 5(b). The calculations assume an average power level of $-78 \mathrm{dBm}$ required at MXC (see Sect. 2.2.2) and also take into account attenuation in the RF cables.

For simplicity we stick to the values for which attenuation in cables has been neglected in the following discussion of $n_{\mathrm{MXC}}$ (solid bars in Fig. 5(a)). We first consider configuration $\mathrm{C} 1=\{0,10,0,20,30\} \mathrm{dB}$, for which we calculate $n_{\mathrm{MXC}} \approx 0.0012$. This is only slightly larger than the lower bound $n_{\mathrm{MXC} \text {,min }} \approx 0.001$ for a total of $60 \mathrm{~dB}$ of attenuation, corresponding to a configuration $C_{\min }=\{0,0,0,0,60\} \mathrm{dB}$, see also Sect. 2.2.1. The reason for the low $n_{\mathrm{MXC}}$ of $\mathrm{C} 1$ is that $n_{\mathrm{MXC}}$ considered as a function of attenuation on each of the stages is in the linear regime for configuration C1, see Fig. 2(a). 

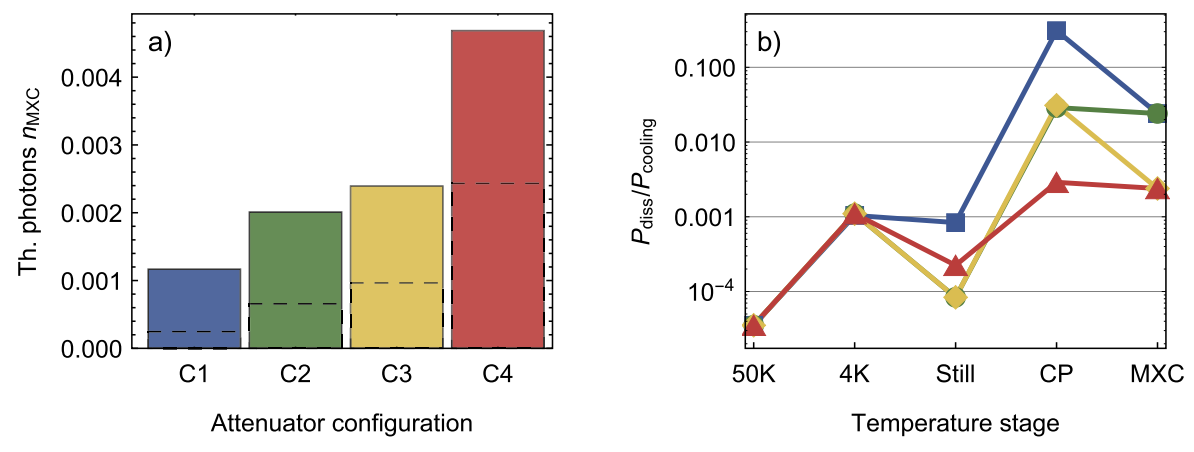

C1: $\{0,10,0,20,30\} d B$

C3: $\{0,20,0,20,20\} d B$

$C 2:\{0,20,0,10,30\} d B$

$C 4:\{0,20,10,10,20\} d B$

Figure 5 Thermal noise photons and signal dissipation. (a) Thermal noise photon number $n_{M X C}$ at $6 \mathrm{GHz}$ for four attenuator configurations C1-C4 in the drive lines (see text). Taking into account attenuation in the cables reduces $n_{\text {MXC }}$ to the values indicated by the dashed bars. (b) Dissipated power divided by cooling power on the indicated stages of the DR due to the operation of 25 drive lines with signal levels translating into an average power level of $-78 \mathrm{dBm}$ at the input of the quantum processor. Calculations for the indicated four attenuator configurations $\mathrm{C1}-\mathrm{C} 4$ are shown

While the relative loads on the $50 \mathrm{~K}, 4 \mathrm{~K}$, and Still stages are negligibly small, the $\mathrm{CP}$ and MXC stages are subject to a significant load.

Since configuration $\mathrm{C} 1$ has a particularly large relative load of $35 \%$ on the $\mathrm{CP}$ stage, we consider configurations with a total of $40 \mathrm{~dB}$ attenuation on the $\mathrm{CP}$ and MXC stages instead of $50 \mathrm{~dB}$. In particular, we target configurations with relative loads below $5 \%$. This is to keep some margin, e.g. for the installation of absorptive infra-red blocking filters, see end of this Section. Removing $10 \mathrm{~dB}$ of attenuation at the $\mathrm{CP}$ and adding $10 \mathrm{~dB}$ at the $4 \mathrm{~K}$ stage instead results in $C 2=\{0,20,0,10,30\} \mathrm{dB}$ and reduces the active load on the $\mathrm{CP}$ by a factor 10 . The resulting value of $n_{\mathrm{MXC}}=0.002$ is almost a factor of two larger than in $\mathrm{C} 1$ because $20 \mathrm{~dB}$ of attenuation at the $4 \mathrm{~K}$ stage already corresponds to the regime where $n_{\mathrm{MXC}}$ as a function of $A_{4 \mathrm{~K}}$ starts to saturate to the $4 \mathrm{~K}$ noise floor, see Fig. 2(a). We also note that dissipation on the $4 \mathrm{~K}$ stage is relatively low compared to the available cooling power and thus redistributing some of the attenuation at $4 \mathrm{~K}$ to the $50 \mathrm{~K}$ stage is not necessary. Configuration $\mathrm{C} 3=\{0,20,0,20,20\} \mathrm{dB}$ corresponds to $\mathrm{C} 2$ with $10 \mathrm{~dB}$ of attenuation moved from the MXC to the $\mathrm{CP}$ stage. In this case the noise photon number only increases by $20 \%$ compared to $\mathrm{C} 2$ to $n_{\mathrm{MXC}}=0.0024$. This is because at $A_{\mathrm{CP}}=20 \mathrm{~dB} n_{\mathrm{MXC}}$ considered as a function of $A_{\mathrm{CP}}$ is still relatively far from reaching the $\mathrm{CP}$ noise floor. At the same time the relative active load on the MXC stage is reduced by a factor of 10 to $<1 \%$.

To further reduce the active load on the $\mathrm{CP}$ by a factor ten one can redistribute $10 \mathrm{~dB}$ from the $\mathrm{CP}$ to the Still, resulting in configuration $C 4=\{0,20,10,10,20\} \mathrm{dB}$. However, $n_{\mathrm{MXC}}$ thereby increases by a factor of two compared to C3, which can be explained with similar arguments as above. A relatively small increase in $n_{\mathrm{MXC}}$ as compared to C3 can be achieved by redistributing only $3 \mathrm{~dB}$ from the $\mathrm{CP}$ to the Still, reducing the load on the $\mathrm{CP}$ by a factor two. However, this is not very practical since it comes along with a non-standard attenuation of $17 \mathrm{~dB}$ at the $\mathrm{CP}$. In addition, solutions with four attenuators require an additional hardware cost of one attenuator and one connectorized RF cable per line as compared to solutions with only three attenuators per line. 
As a compromise between a low number of noise photons and a low heat load on MXC we select $\mathrm{C} 3$, with a noise photon number of about 0.001 (taking attenuation in cables into account).

To filter infrared radiation that can lead to quasi-particle generation and thus to a reduced $T_{1}$ time [8-13], we provide the option to install a custom-built, IR filter based on Eccosorb CR-124 absorber material right before the attenuator at MXC, see Appendix 3. With an attenuation of $6 \mathrm{~dB}$ at $6 \mathrm{GHz}$, it further reduces $n_{\mathrm{MXC}}$ by a factor four, while increasing dissipation on all stages by a factor four due to the larger power required at the input of the cryostat to achieve the same signal level at MXC.

\subsection{Flux line configuration}

To reduce current and thus magnetic flux noise in the SQUID loop of a qubit, a suitable amount of attenuation needs to be installed in the flux lines. Part of this attenuation can be realized at room temperature to reduce $1 / \mathrm{f}$ noise and white noise from the instruments connected to the flux line (e.g. an arbitrary waveform generator) to the level of room temperature Johnson-Nyquist noise. To reduce the noise level below room temperature Johnson-Nyquist noise we install a single attenuator $A_{4 \mathrm{~K}}$ at the $4 \mathrm{~K}$ stage. The current-noise power spectral density after this attenuator is calculated as

$$
S_{I}^{\text {th, eff }}=\frac{S_{I}^{\text {th }}\left(T_{\mathrm{RT}}\right)}{A_{4 \mathrm{~K}}}+\frac{A_{4 \mathrm{~K}}-1}{A_{4 \mathrm{~K}}} S_{I}^{\text {th }}\left(T_{4 \mathrm{~K}}\right)
$$

where $S_{I}^{\text {th }}(T)=2 \mathrm{k}_{\mathrm{B}} T / R$ is the two-sided current-noise power spectral density of JohnsonNyquist noise. The resistance $R=50 \Omega$ corresponds to the internal impedance of a typical arbitrary waveform generator. Note that as opposed to using Eq. (2) in the context of thermal noise in the drive lines, considering the classical Johnson-Nyquist formula here is sufficient since the thermal energy at $T_{4 \mathrm{~K}} \approx 3 \mathrm{~K}$ is much larger than the relevant noise frequencies of $\lesssim 1 \mathrm{GHz}$. We plot $S_{I}^{\text {th,eff }}$ as a function of $A_{4 \mathrm{~K}}$ for $T_{\mathrm{RT}} \approx 300 \mathrm{~K}$ and $T_{4 \mathrm{~K}} \approx 3 \mathrm{~K}$ in Fig. 2(b). Similar to Fig. 2(a) $S_{I}^{\text {th,eff }}$ saturates at the thermal noise floor of the $4 \mathrm{~K}$ stage for attenuator values $A_{4 \mathrm{~K}} \gtrsim 20 \mathrm{~dB}$.

We use $S_{I}^{\text {th,eff }}$ for deriving upper bounds on the coherence times $T_{2}^{*}$ and the echo coherence time $T_{2}$ due to thermal noise in the flux lines $[7,16]$

$$
T_{2}^{*}=\frac{1}{2} T_{2}^{E}=\frac{2}{D^{2} S_{I}}
$$

Here, $S_{I}(\omega)=\int \mathrm{d} t\langle\delta I(0) \delta I(t)\rangle \mathrm{e}^{-i \omega t}$ is the power spectral density of the current noise and $D$ the sensitivity of the qubit transition frequency $\omega_{\mathrm{q}}$ to the current $I$ in the flux line

$$
D=\frac{\partial \omega_{\mathrm{q}}}{\partial I}=\frac{\partial \omega_{\mathrm{q}}}{\partial \Phi} \frac{\partial \Phi}{\partial I} .
$$

Using a typical mutual inductance between flux line and SQUID loop of $M=\frac{\partial \Phi}{\partial I}=$ $0.5 \Phi_{0} / \mathrm{mA}$ we obtain $T_{2}^{*}=(46,426,2333) \mu \mathrm{s}$ for an attenuation of $(0,10,20) \mathrm{dB}$ at a $10 \%$ detuning from the sweet spot frequency of a qubit with a symmetric SQUID loop [19]. We thus conclude that an attenuation of $10-20 \mathrm{~dB}$ at the $4 \mathrm{~K}$ stage is sufficient to mitigate qubit dephasing due to thermal noise. Noise from electronic instruments connected to 
the flux line should be reduced to the level of Johnson-Nyquist noise by installing a suitable amount of attenuation at room temperature outside of the cryostat. Other sources of magnetic flux noise include surface adsorbates and defects [45-47] and external magnetic field fluctuations in combination with insufficient magnetic shielding.

Since the bandwidth of typical flux pulses is at most $1 \mathrm{GHz}[21,22,42]$, we install a lowpass filter with a $3 \mathrm{~dB}$ bandwith of about $1.9 \mathrm{GHz}$ (Minicircuits Minicircuits VLFX1300+) in the flux line to suppress thermal noise photons at qubit frequencies, which could otherwise couple to the qubit due to parasitic capacitive or inductive coupling of the on-chip flux line and the qubit. A custom-built, absorptive IR filter is installed with the goal to reduce quasi-particle generation in the superconducting film, see above and Appendix 3 for details. It is mounted in the RF lines at the MXC stage to achieve the lowest infrared noise photon number.

\subsection{Output line configuration}

We designed four output lines for the detection of qubit readout signals on the single photon level [28, 48]. We use travelling wave Josephson parametric amplifiers (TWPA) [49] as near-quantum limited amplifiers installed at MXC, see Fig. 6(a). Their near-quantum
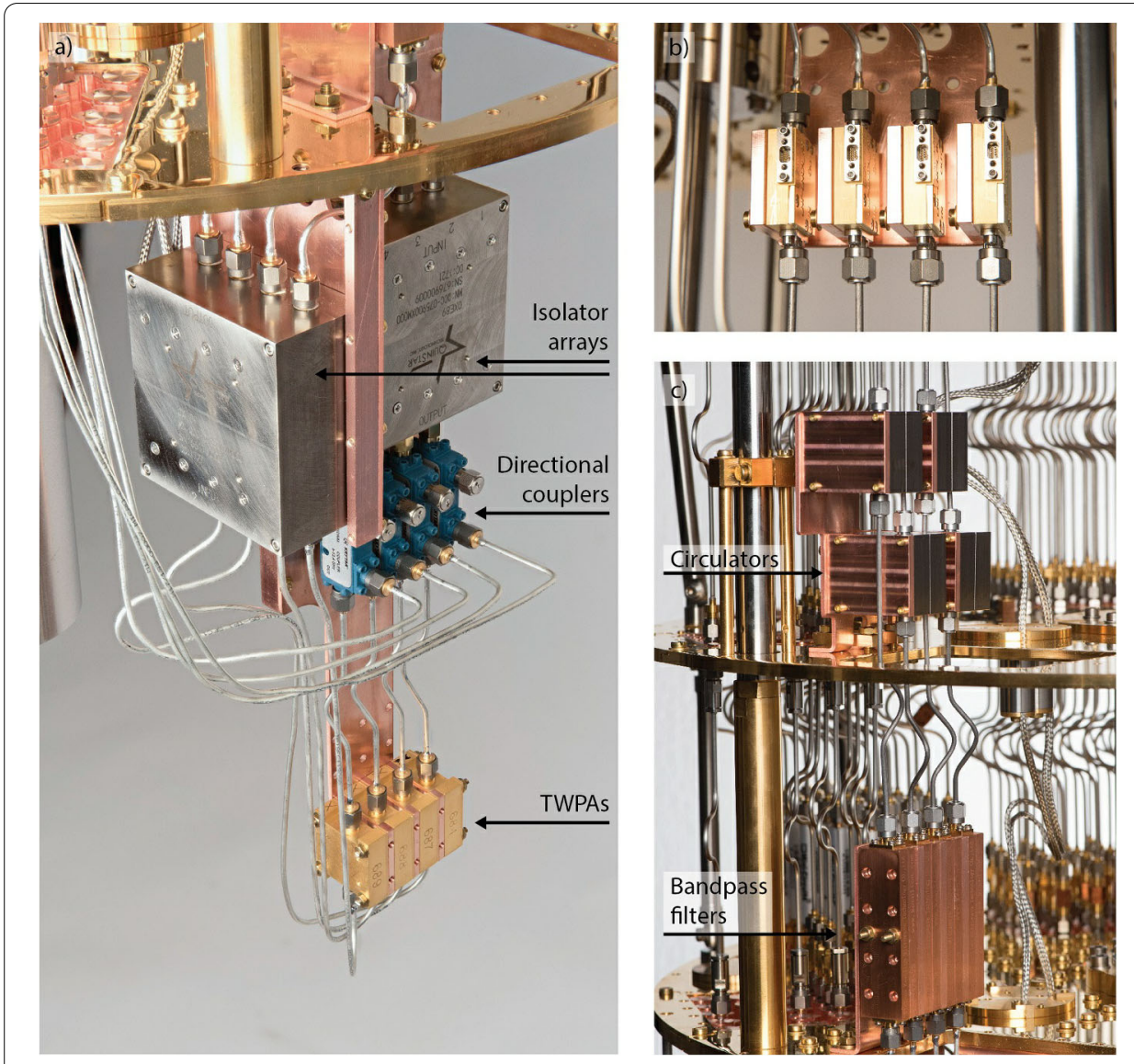

Figure 6 Integration of four high-bandwidth output lines. (a) Components mounted on the MXC plate: readout signals from the quantum processor travel through a first 4-channel, magnetically shielded isolator array (right), followed by directional couplers, TWPAs and a second, identical isolator array (left). (b) Four HEMT amplifiers mounted on the $4 \mathrm{~K}$ plate. (c) Four bandpass filters, mounted and thermalized at the MXC plate, followed by four circulators mounted on the cold plate 
limited performance together with their broadband gain of about 20-30 dB over a frequency range of 3-12 GHz allow for fast single-shot readout with frequency multiplexing [50], where about ten qubits can be simultaneously read out using a single output line. High-electron mobility transistor (HEMT) amplifiers (LNF LNC4_8C) with a gain of about $40 \mathrm{~dB}$ are installed at the $4 \mathrm{~K}$ stage, see Fig. 6(b). More than a total of $60 \mathrm{~dB}$ isolation towards the sample is provided by a circulator (Quinstar QCY-060400CM00) at CP and two isolators at MXC. The two isolators with an operation range of 3-12 $\mathrm{GHz}$ are also required for impedance matching of the TWPA over its entire gain bandwidth. For this purpose, one of the isolators is installed before and one after the TWPA, see Fig. 3(b). Each isolator is part of a four-channel isolator array (Quinstar QCC-075900XM000), see Fig. 6(a). Circulators and isolators are both magnetically shielded.

A reflective bandpass filter (Keenlion KBF-4/8-2S) at MXC suppresses noise at frequencies outside the bandwidths of circulator and isolators, see Fig. 6(c). To minimize signal loss between the two amplfiers, we use superconducting NbTi coaxial cables between MXC and $4 \mathrm{~K}$. To minimize passive heat load we chose stainless steel cables from $4 \mathrm{~K}$ to room temperature. According to Friis formula the increased loss compared to e.g. SPCW cable does not decrease the signal to noise ratio of the amplifier chain. E.g. a loss of $10 \mathrm{~dB}$ in the cable section between HEMT and the first amplifier at room temperature can be tolerated if one uses a low noise amplifier with a noise temperature of about $500 \mathrm{~K}$ as a first amplifier at room temperature. ${ }^{\mathrm{d}}$

For the operation of the TWPA a microwave pump signal with a typical frequency in the range $6-8 \mathrm{GHz}$ and a power level at the input of the TWPA of about $-55 \mathrm{dBm}$ is required. This pump signal is added to the TWPA through the isolated port of a $20 \mathrm{~dB}$ directional coupler $^{\mathrm{e}}$ (Krytar 120420), see Fig. 3(b). To realize the relatively large pump power we use pump lines with a reduced total attenuation of $50 \mathrm{~dB}$ attenuation as compared to the $60 \mathrm{~dB}$ attenuation used in the drive lines. This $50 \mathrm{~dB}$ of attenuation includes the $20 \mathrm{~dB}$ attenuation provided by the directional coupler with its coupled port terminated with a $50 \Omega$ load resistance. Photographs of the readout components described in this Section are shown in Fig. 6. The TWPAs and the isolator arrays are mounted on $2 \mathrm{~mm}$ thick $\mathrm{Cu}$ sheets whose stability is enforced by $\mathrm{Cu}$ bars at their edges, see Fig. 6(a). For compact mounting and thermalization, the four TWPAs and the four bandpass filters (Fig. 6(c)) are first stacked using $\mathrm{Cu}$ spacers, which are then mounted to the $\mathrm{Cu}$ sheet or to an L-shaped bracket, respectively. Similarly, the four HEMT amplifiers and the four circulators are first mounted onto $\mathrm{Cu}$ pieces which are then mounted to a common bracket.

\section{Direct measurements of the passive heat load}

To determine the passive heat load induced by each cable tree and to extract a heat load per line, we performed reference measurements of the cooling power in the dilution refrigerator system as delivered without microwave cabling. Then we cooled down the system each time we installed a new cable tree and recorded the temperature increase on each of the stages of the dilution refrigerator to infer the corresponding passive heat loads. The cooldown of cable trees comprising up to 25 identical lines of a given type has the advantage of generating temperature increases which are larger than those created by a single line and also larger than typical run-to-run temperature variations on the stages of the dilution refrigerator. Furthermore, this method naturally averages over variations in material and components, as well as over possible variations occurring in the installation and thermalization of individual cables and attenuators. 


\subsection{Reference measurements}

Our reference measurements consist of applying a well defined electrically created heat load to a given temperature stage and recording the steady state temperature increases on all other stages. In steady state, the applied heat load corresponds to the cooling power on that stage at its steady state temperature. The used temperature sensors and heaters are described in Appendix 4. The measured relative temperature increases are shown as a function of heat load applied to a given stage in Fig. 7. The absolute temperature increase on the stage to which the power is applied is indicated on the right axis of each plot. The $50 \mathrm{~K}$ stage and the $4 \mathrm{~K}$ stage show approximately linear temperature increases over the range of applied powers (up to $10 \mathrm{~W}$ for the $50 \mathrm{~K}$ stage and $0.9 \mathrm{~W}$ for the $4 \mathrm{~K}$ stage). Data with a larger range of applied powers is presented in Appendix 5. Heating a given stage also affects stages at lower temperature because they are thermally connected via structural elements of the dilution refrigerator such as posts and other elements such as the dilution unit and pre-installed DC wiring. In addition the ${ }^{3} \mathrm{He}$ reaching the Still in the gas phase for condensation is effectively hotter when $50 \mathrm{~K}$ and $4 \mathrm{~K}$ stages are heated leading to a larger load on the Still.

The cooling power on the Still stage is provided mainly by the evaporation of ${ }^{3} \mathrm{He}$ and reflects the dependence of the partial pressure of ${ }^{3} \mathrm{He}$ on temperature [51, 52]. The temperature of the Still plate increases by about $60 \%$ over the range of applied heat powers of
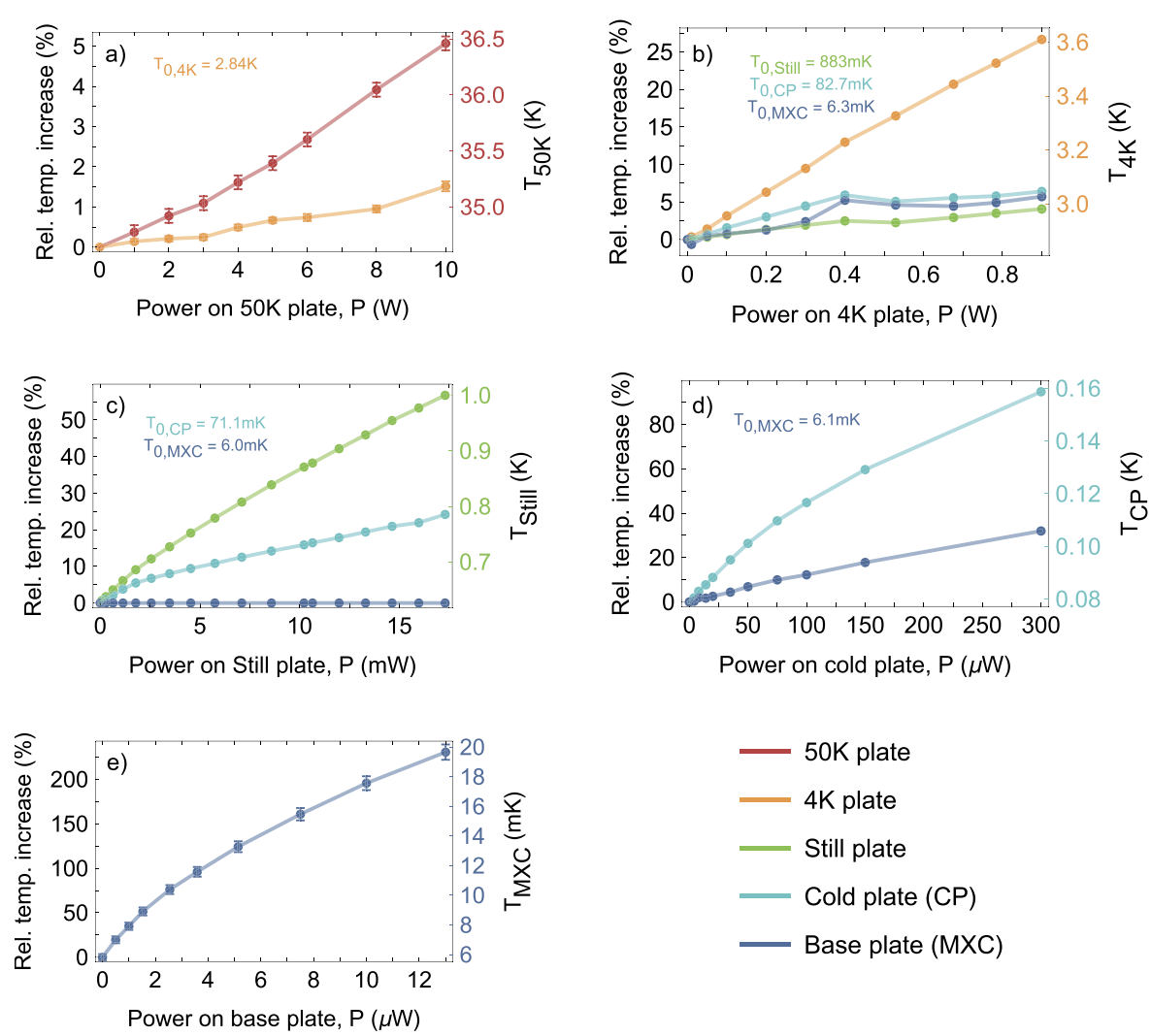

- $50 \mathrm{~K}$ plate

— $4 \mathrm{~K}$ plate

_ Still plate

- Cold plate (CP)

Base plate $(\mathrm{MXC})$

Figure 7 Reference measurements in the DR system as delivered. Temperature increases on the different plates of the DR as a function of applied power to the (a) $50 \mathrm{~K}$, (b) $4 \mathrm{~K}$, (c) Still, (d) CP, (e) MXC stages. The temperatures indicated in the upper left corner of the plots represent the starting temperatures, without applied heat 
0-18 mW, see green data set in Fig. 7(c). Importantly, the heat power applied to the Still stage also sets the cooling power on MXC, $P_{\text {MXC }} \propto \dot{n}_{3} T_{\text {MXC }}^{2}$ [51] because it regulates the flow $\dot{n}_{3}$ of the ${ }^{3} \mathrm{He}$ through the dilution unit and thus across the phase boundary between the concentrated and dilute phases of ${ }^{3} \mathrm{He}$ in the mixing chamber. Since an increased Still temperature also creates an additional load on the CP and MXC stages, there is an optimum flow value which maximizes the cooling power for a given $T_{\mathrm{MXC}}$. The $T_{\mathrm{MXC}}^{2}$ dependence of $P_{\mathrm{MXC}}$ arises because the enthalpy difference between the two phases is proportional to $T_{\mathrm{MXC}}^{2}[51]$. For all measurements we set $P_{\text {Still }}=10.7 \mathrm{~mW}$, corresponding to a flow of $0.69(1) \mathrm{mmol} / \mathrm{s}$ and a cooling power on MXC of about $5 \mu \mathrm{W}(13 \mu \mathrm{W})$ at $13 \mathrm{mK}$ $(20 \mathrm{mK})$, see Fig. 7(e). Importantly, when increasing the flow to $1.0 \mathrm{mmol} / \mathrm{s}$ by applying a heat power of $40 \mathrm{~mW}$ to the Still we reach a cooling power of $19 \mu \mathrm{W}(540 \mu \mathrm{W})$ at $20 \mathrm{mK}$ (100 mK).

The CP has no active cooling element, but since it is mounted on top of the last set of heat exchangers [52] it is effectively cooled by the cold mixture entering and leaving the mixing chamber. We measure the temperature increase over a relatively wide range of heat powers up to $300 \mu \mathrm{W}$ since our attenuator configuration C3 in the drive lines is expected to create a significant active load on the $\mathrm{CP}$. Over this range the temperature of the $\mathrm{CP}$ doubles from about $80 \mathrm{mK}$ to about $160 \mathrm{mK}$, see turquoise data set in Fig. 7(d). The effect on $T_{\mathrm{MXC}}$ is relatively small, e.g. at the largest measured value of $P_{\mathrm{CP}}=300 \mu \mathrm{W} T_{\mathrm{MXC}}$ rises from $6.1 \mathrm{mK}$ to $8.1 \mathrm{mK}$, corresponding to an effective load of only $1 \mu \mathrm{W}$ on the MXC stage.

\subsection{Determination of passive heat loads}

The reference measurements presented in Fig. 7 allow us to convert an increase in temperature $\Delta T_{i}$ observed on stage $i$ after the cooldown of a given cable tree, into a passive heat load

$$
\Delta P_{i}=\frac{\partial P_{i}}{\partial T_{i}} \Delta T_{i}
$$

on that stage. The coefficients $\frac{\partial P_{i}}{\partial T_{i}}$ are extracted from a linear fit to the low temperature regimes of the data in Fig. 7. All measured $\Delta T_{i}$ are sufficiently small to justify the use of this linear expansion. Due to an increased temperature of the next higher stage $i-1$, $\Delta P_{i}$ also contains a small contribution $\left(\partial P_{i} / \partial T_{i-1}\right) \Delta T_{i-1}$ from all elements other than the cables which is not taken into account in the measured steady state temperatures of the dilution refrigerator as delivered. We thus subtract this contribution from $\Delta P_{i}$ to obtain the pure passive heat loads $\Delta \tilde{P}_{i}=\Delta P_{i}-\left(\partial P_{i} / \partial T_{i-1}\right) \Delta T_{i-1}$ of the installed cables. A significant correction $>20 \%$ results only for $\Delta \tilde{P}_{\text {Still }}$ though. We verified this procedure by measuring $T_{i}$ vs. $P_{i}$ curves analogous to the data shown in Fig. 7, but for $P_{i-1} \neq 0$, see Appendix 6 .

Additional care needs to be taken when determining $\Delta T_{\mathrm{CP}}$ and $\Delta T_{\mathrm{MXC}}$. This is because an increased Still temperature changes the flow through the dilution unit thereby not only leading to an additional passive load on $\mathrm{CP}$ and $\mathrm{MXC}$, but effectively changing the cooling power vs. temperature curves. This is most evident for the MXC stage when considering the relation $P_{\mathrm{MXC}} \propto \dot{n}_{3} T_{\mathrm{MXC}}^{2}$ discussed above. Before determining $\Delta T_{\mathrm{CP}}$ and $\Delta T_{\mathrm{MXC}}$ we thus reduce the Still heat power such that we reach $T_{\text {Still }}=882(1) \mathrm{mK}$, resulting in the same flow of 0.69(1) $\mathrm{mmol} / \mathrm{s}$ set for the reference measurements. 
Table 2 Passive heat load per line. Passive heat load $(\mathrm{HL})$ of cable types installed in the DR, as inferred from observed temperature increases after the installation of individual cable trees into the DR. The upper and middle sections of the table refer to $0.085^{\prime \prime}$ diameter stainless steel coaxial cable (UT-085-SS-SS) with attenuator configurations used in drive lines and flux lines, respectively. Indicated errors include statistical errors between different cooldowns and reflect run-to-run temperature variations on the stages of the DR. The intervals of estimated heat loads correspond to calculations of lower and upper bounds (see text for details)

\begin{tabular}{llllll}
\hline & $50 \mathrm{~K}$ & $4 \mathrm{~K}$ & \multicolumn{1}{c}{ Still } & $\mathrm{CP}$ & $\mathrm{MXC}$ \\
\hline Drive line UT-085-SS-SS & & & & & \\
$\quad$ Measured HL & $45(34) \mathrm{mW}$ & $1.0(5) \mathrm{mW}$ & $4(3) \mu \mathrm{W}$ & $0.4(2) \mu \mathrm{W}$ & $0.013(6) \mu \mathrm{W}$ \\
$\quad$ Estimated HL & $24-27 \mathrm{~mW}$ & $0.4-1.9 \mathrm{~mW}$ & $1.6-2.1 \mu \mathrm{W}$ & $0.33-0.60 \mu \mathrm{W}$ & $0.004 \mu \mathrm{W}$ \\
Flux line UT-085-SS-SS & & & & & \\
$\quad$ & & & & \\
$\quad$ Measured HL & $56(39) \mathrm{mW}$ & $1.2(8) \mathrm{mW}$ & $2(1) \mu \mathrm{W}$ & $0.3(1) \mu \mathrm{W}$ & $0.029(5) \mu \mathrm{W}$ \\
$\quad$ Estimated HL & $24-27 \mathrm{~mW}$ & $0.4-1.9 \mathrm{~mW}$ & $1.6-2.1 \mu \mathrm{W}$ & $0.24-0.33 \mu \mathrm{W}$ & $0.005-0.282 \mu \mathrm{W}$ \\
Output line UT-085-NbTi & & & & & \\
$\quad$ & - & - & - & $0.3(3) \mu \mathrm{W}$ & $0.020(16) \mu \mathrm{W}$ \\
$\quad$ Estimated HL & - & - & - & $0.18-0.31 \mu \mathrm{W}$ & $0.002-0.322 \mu \mathrm{W}$ \\
\hline
\end{tabular}

To extract the passive heat loads $\Delta \tilde{P}_{i}$ for each type of line we cooled down the dilution refrigerator after individual installation of the drive line cable tree, the readout cable tree without the NbTi lines, the four NbTi output lines between the $4 \mathrm{~K}$ stage and the MXC stage, and the flux line cable tree, respectively. From the extracted total passive heat load after each cooldown we calculate an average load per line by dividing the total loads by the number of lines in the cable tree.

To extract a mean passive load per drive line we combine the data from the cooldown of the drive line tree and the readout tree since the readout tree consists of drive lines and pump lines having the same thermalization scheme with attenuators at the $4 \mathrm{~K}, \mathrm{CP}$, and MXC stages. The data is shown in the first section of Table 2. Variations in both data sets are of the order of typical run-to-run temperature variations and therefore make up for about half of the indicated errors. The determined heat loads are in qualitative agreement with calculated values based on Eq. (1), see row "Estimated HL" in the upper part of Table 2. The lower and upper bounds of the intervals represent estimates in which thermalization of the center conductor and dielectric on stages without attenuator $(50 \mathrm{~K}$ and Still stage) is fully achieved or in which the center conductor and dielectric are not thermalized at all on these stages. ${ }^{f}$ In calculating the predicted passive load we have assumed that the center conductor and dielectric are fully thermalized on stages with an attenuator. However, it is an open question how well the center conductor thermalizes in an attenuator and it is likely to depend on the physical structure and thermal properties of the attenuator [4].

While the measured loads on the $50 \mathrm{~K}, 4 \mathrm{~K}$, Still, CP stages agree within error bars with the estimated values, the load on the MXC stage is larger than predicted. This can have several reasons. First, there could be discrepancies between the thermal conductivitiy of the stainless steel (SS) of the cables and the data used for the estimates in that temperature interval. Second, thermalization of the center conductor via the attenuator at the $\mathrm{CP}$ could be incomplete. Third, the long timescales for thermalization of components at MXC, in particular if they contain hydrogen [53], can lead to an overestimation of the heat load since we measure $\Delta T_{i}$ always at the same time ( $\sim 1$ day after the condensation of the mixture when the MXC temperature is sufficiently stable). Fourth, gas desorbing 
from newly installed components may freeze out at MXC, creating an additional heat load.

The average passive loads per flux line on the $50 \mathrm{~K}, 4 \mathrm{~K}$, and Still stages agree within error bars with the corresponding passive loads per drive line, see second section of Table 2 . This is expected since the two types of lines are nominally identical from the vacuum flange to the Still stage. The load on the CP is comparable to the corresponding load per drive line. This observation is expected because the main contribution to this load originates from the outer conductor. The intervals for the estimated loads are calculated for complete thermalization of the center conductor and dielectric at the $50 \mathrm{~K}$, Still and CP stages or no thermalization of the center conductor and dielectric on these stages at all. We exclude the case in which the center conductor and dielectric thermalize on the CP but not on the Still because the thermal contact resistance increases with decreasing temperature. The measured passive load of 0.029(5) $\mu \mathrm{W}$ per flux line on MXC is more than a factor of two larger than the passive load per drive line. We explain this observation by the absence of an attenuator in the flux line at the CP causing a direct passive load from the Still to the MXC stage via the center conductor and dielectric. The estimated upper bound of $0.282 \mu \mathrm{W}$ is calculated by assuming no thermalization of the center conductor and dielectric on neither the CP nor the Still stage. Since this upper bound is by a factor of ten larger than the measured value, it is likely that the center conductor and dielectric thermalize at the Still stage. In this case the estimated load amounts to $0.054 \mu \mathrm{W}$.

The passive loads extracted from the cooldown of the four $\mathrm{NbTi}$ lines are listed in the third section of Table 2. These loads lie within the estimated lower and upper bounds corresponding to complete thermalization of the center conductor and dielectric at Still and $\mathrm{CP}$ or no thermalization at all, respectively. Using an argument analogous to the one used for the flux lines, it is likely that the center conductor and dielectric thermalize at the Still stage. How well the center conductor and dielectric thermalize at the CP cannot be evaluated given the size of the error bars of the extracted passive loads.

In summary, after the installation of 66 RF lines, mainly UT-085-SS-SS, $T_{\mathrm{MXC}}$ increased from 6.1(1) $\mathrm{mK}$ to 8.4(2) $\mathrm{mK}$, corresponding to a total passive heat load on MXC of 1.4(2) $\mu \mathrm{W}$. This is more than a factor of ten smaller than the available cooling power at $20 \mathrm{mK}$, and thus sets the stage for experiments with tens to hundreds of superconducting qubits.

\section{Active load measurements}

To determine the active load during the operation of a quantum processor we apply continuous signals of a given power to a single drive line or current to a single flux line, and measure the resulting heat loads on each of the stages of the DR. Estimating typical duty cycles and power levels discussed in Sect. 2.2 allows us to estimate the total active load from the measured loads.

\subsection{Drive lines}

To experimentally test loads due to signal dissipation in drive lines we apply a RF signal to one of them and measure the corresponding temperature increases on the stages in the dilution refrigerator. Several drive lines were tested and their results agreed within error bars. In particular, we attempt to evaluate whether the power dissipated in an attenuator predominantly flows to the stage on which the attenuator is thermalized, or whether 
part of that heat flows to the next lower stage. This is important because the signal levels at stages with attenuators differ by $20 \mathrm{~dB}$ in the selected attenuator configuration of $C 3=\{0,20,0,20,20\} \mathrm{dB}$. In other words, the power dissipated in an attenuator at the $4 \mathrm{~K}$ (CP) stage is by a factor 100 larger than the power dissipated in the attenuator at the $\mathrm{CP}$ (MXC) stage. Hence, a small fraction of heat dissipated in an attenuator at a given stage flowing towards the lower temperature stages can significantly increase the heat load on those stages.

We first apply a power of $10 \mathrm{dBm}$ at a frequency of $10 \mathrm{MHz}$, at which dissipation in the $\mathrm{RF}$ cables themselves is negligible. This allows for a simple comparison with the expected loads of $10 \mathrm{~mW}$ on the $4 \mathrm{~K}$ stage, $100 \mu \mathrm{W}$ on CP, and $1 \mu \mathrm{W}$ on MXC. Using our reference measurements, we extract an active load of $11.5(1.6) \mathrm{mW}$ on the $4 \mathrm{~K}$ stage, 95(9) $\mu \mathrm{W}$ on $\mathrm{CP}$, 1.1(1) $\mu \mathrm{W}$ on MXC, in agreement with the predicted values. On the Still stage we measure an active load of 48(6) $\mu \mathrm{W}$, which we attribute to a small fraction of power dissipated at $4 \mathrm{~K}$ (about $0.4 \%$ ) flowing towards the Still stage. Our measurements show that more than $99 \%$ of the power dissipated in an attenuator flows to the stage on which the attenuator is anchored, thereby validating our method for thermalizing attenuators. We note that the extracted active loads represent pure active loads, i.e. we subtract from the measured load on a given stage the additional passive load due to an increased temperature of the next higher stage.

In addition, we applied a microwave tone at $5 \mathrm{GHz}$ with a power of $16 \mathrm{dBm}$ resulting in well measurable temperature increases on the lower stages. To calculate the power level at MXC, we have to take into account the attenuation in the cables [36], as discussed in Appendix 2. The total attenuation in the cables sums up to about $8.5 \mathrm{~dB}$, and with the $60 \mathrm{~dB}$ of discrete attenuation yields a signal level at MXC of about $-52.5 \mathrm{dBm}$, much larger than the average value of $-78 \mathrm{dBm}$ required in a drive line, see Sect. 2.2.2. Hence, to predict an active load per drive line the extracted pure active loads shown as blue rectangles in Fig. 8 need to be scaled by $-78 \mathrm{dBm} /-52.5 \mathrm{dBm}=-25.5 \mathrm{~dB}$. On stages with attenuators the data agrees well with the estimated active loads (red triangles). Error bars correspond to whether we assign the dissipated power in a cable section itself (excluding the attenuators) to the stage above or to the stage below. On the Still stage the measured load is by a factor two larger than estimated, which is consistent with the above observation made at a signal frequency of $10 \mathrm{MHz}$. Our measurements show that active load in drive lines is

Figure 8 Dissipation in drive lines. Dissipated power at the temperature stages of the DR when applying a microwave tone at $5 \mathrm{GHz}$ and a power of $16 \mathrm{dBm}$, corresponding to a signal level of $-52.5 \mathrm{dBm}$ at MXC. Shown are measured data (blue rectangles, solid line) and results of calculations (red triangles, dashed line)

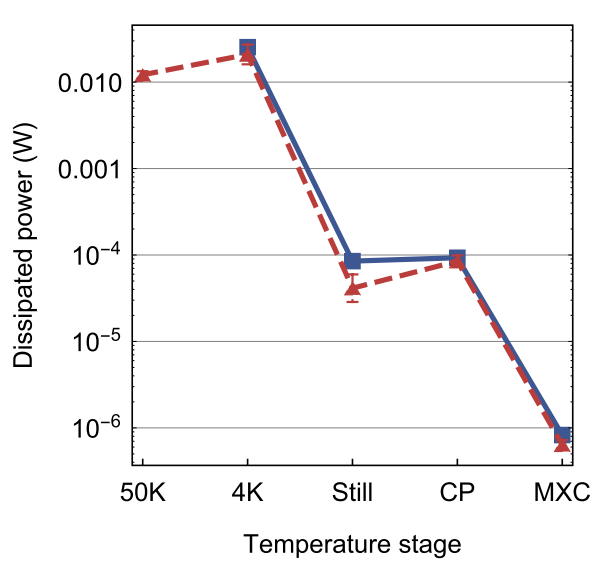


well captured by assigning the power dissipated in an attenuator to the stage on which it is thermalized.

We also measured the active load per TWPA pump line and found values comparable to the active load per drive line. This results from the fact that the required pump power at the input of the TWPA is with about $-65 \mathrm{~dB}$ by $13 \mathrm{~dB}$ larger than the required average power in a drive line but the TWPA pump lines having $10 \mathrm{~dB}$ less attenuation than the drive lines, see Fig. 3(b). The largest relative active load of a pump line arises at the $\mathrm{CP}$ stage and amounts to $0.1 \%$, corresponding to $0.02 \mu \mathrm{W}$ in absolute numbers.

In this section, we discussed the experimentally determined active loads for the selected attenuator configuration $\{0,20,0,20,20\} \mathrm{dB}$ in the drive lines. Since this configuration has been chosen to keep the active load significantly below the available cooling powers, the active load due to execution of single-qubit gates has only a small influence on the temperature of the quantum processor. Whether the thermal photon number is as low as predicted needs to be experimentally determined $[3,4]$ and would provide information about the effective temperatures at which the lowest temperature attenuators emit blackbody radiation.

\subsection{Flux lines}

To determine the active load arising from flux biasing of the qubits, we terminate one of the flux lines at MXC with an electric short, apply a DC current to it, and measure the corresponding temperature increase and heat loads. While the relative temperature increases are negligibly small on the stages from room temperature to the Still, they are significant on CP and MXC. As expected we observe a quadratic increase of the extracted loads $P_{\mathrm{MXC} / \mathrm{CP}}=R_{\mathrm{eff}, \mathrm{MXC} / \mathrm{CP}} I_{\mathrm{MXC}}^{2}$ as a function of the applied current, see Fig. 9. The maximum applied current of $8.8 \mathrm{~mA}$ at the input of the dilution refrigerator results in $4.6 \mathrm{~mA}$ after the $10 \mathrm{~dB}$ attenuator at $4 \mathrm{~K}$, and thus at MXC (denoted $I_{\mathrm{MXC}}$ ). From quadratic fits to the data we extract $R_{\mathrm{eff}, \mathrm{MXC}}=$ $0.15(1) \Omega=0.15(1) \mu \mathrm{W} / \mathrm{mA}^{2}$ and $R_{\text {eff,CP }}=0.42(2) \Omega$. The sum of $R_{\text {eff,MXC }}$ and $R_{\text {eff,CP }}$ corresponds to the resistance of a coaxial stainless steel cable with an effective length $L_{\text {eff }}=20 \mathrm{~cm}$. For comparison, the cable length between Still and CP (CP and MXC) amounts to $19 \mathrm{~cm}(11 \mathrm{~cm})$. To estimate $L_{\text {eff }}$ a priori one would need to know details such as the thermal conductances between the center conductor, which contributes most to the resistance of the coaxial cable, and the stages of the dilution refrigerator.
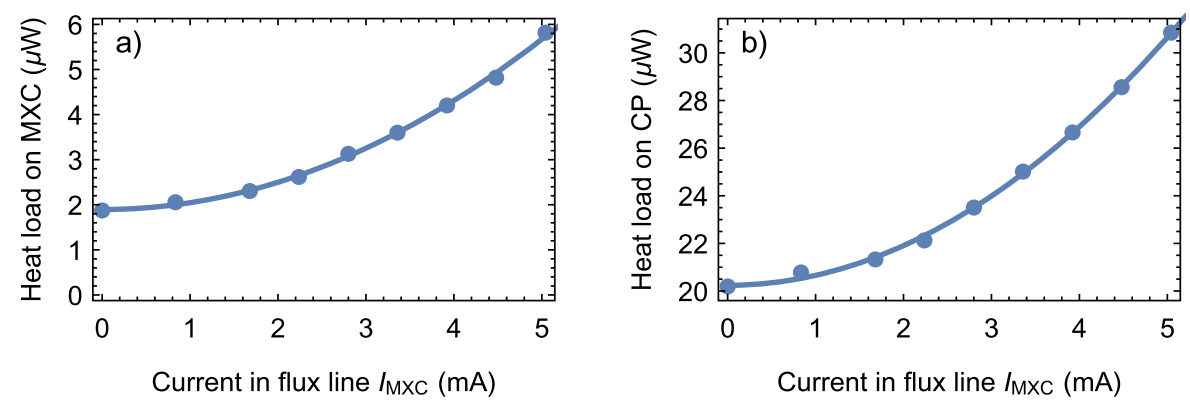

Figure 9 Dissipation in flux lines. Dissipated power at MXC (left) and CP (right) measured as a function of current in the flux line. Solid lines are quadratic fits to the data 


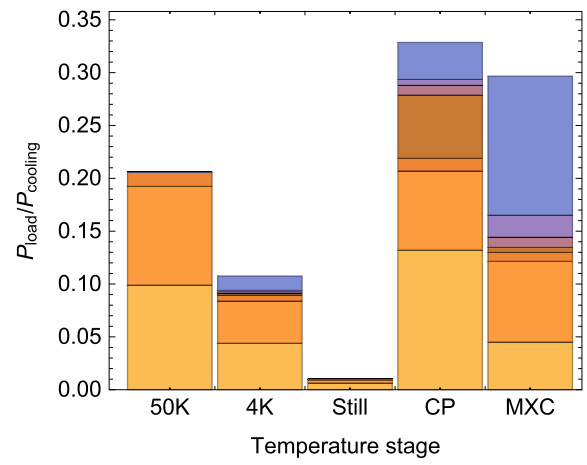

50x active load flux line, DC flux biasing

50x active load flux line, flux pulses

$8 x$ active load pump line

50x active load drive line

$8 x$ passive load output line

50x passive load flux line

50x passive load drive line

$8 x$ passive load read-in line

8x passive load pump line

Figure 10 Total heat load budget for the operation of a 50 qubit processor. Predicted passive and active heat loads for the operation of a 50 qubit processor with individual drive and flux control. The heat loads are normalized to the measured cooling powers as presented in Table 1

For a reasonable choice of mutual inductance between flux line and qubit discussed in Sect. 2.2.2, the DC biasing currents needed to tune the qubits to the desired transition frequencies are considered to be randomly distributed in the interval $\left[-I_{\max }, I_{\max }\right]=$ $[-1,1] \mathrm{mA}$. Hence, an average power of $P_{\mathrm{avg}}=\left(1 / I_{\mathrm{max}}\right) \int_{0}^{I_{\max }} R_{\mathrm{eff}} I_{\mathrm{MXC}}^{2} \mathrm{~d} I_{\mathrm{MXC}}=\frac{1}{3} R_{\mathrm{eff}} I_{\max }^{2}$ is dissipated, evaluating to $0.050(3) \mu \mathrm{W}(0.140(7) \mu \mathrm{W})$ on MXC (CP). For 25 flux lines this sums up to 1.25(7) $\mu \mathrm{W}(3.5(2) \mu \mathrm{W})$ on MXC (CP), which is about a factor of 15 (40) smaller than the cooling power available at MXC (CP) at a temperature of $20 \mathrm{mK}(140 \mathrm{mK})$. We note that this is a worst-case scenario since with careful magnetic shielding it should be possible to reduce the offset fluxes to values close to zero.

For an estimate of dissipation due to flux pulses used for two-qubit gates we use the measured effective DC resistances, the required current amplitude of about $0.4 \mathrm{~mA}$ and a duty cycle of 33\% (see Sect. 2.2.2). The resulting heat loads on CP and MXC are then evaluated to $0.20(1) \mu \mathrm{W}(0.55(3) \mu \mathrm{W})$ on MXC (CP), corresponding to about one sixth of the corresponding loads due to DC flux biasing, see Fig. 10.

\section{Conclusion and outlook}

Based on the presented measurements of passive and active loads we estimate the total heat load acting on the different stages of a dilution refrigerator, see Fig. 10, when operating a 50 qubit processor with individual drive and flux control and with a multiplexed readout architecture allowing for simultaneous readout of sets of 6-7 qubits. Such a quantum processor requires a total of $124 \mathrm{RF}$ lines ( 50 drive lines, 50 flux lines, 8 output lines, 8 readout resonator drive lines, 8 TWPA pump lines), corresponding to the operation of the presented system at its full capacity. The largest relative loads of about $30 \%$ occur at the $\mathrm{CP}$ and MXC stages, with about an equal share between passive and active loads. The load on MXC corresponds to an operation temperature of $14 \mathrm{mK}$. The active load in the drive lines is a result of targeting a noise photon number of $10^{-3}$ or less. The relative loads of about $20 \%$ and $10 \%$ on the $50 \mathrm{~K}$ and $4 \mathrm{~K}$ stages, respectively, are dominated by passive loads. The load on the Still stage is negligibly small. Hence, the thermal performance of the discussed system would allow for the operation of a 150 qubit processor if the capacity for hosting coaxial lines was increased three-fold. This capacity increase could be achieved either by increasing the density of coaxial line integration or by building a larger diameter dilution refrigerator. 
The material of the coaxial cables has been chosen to minimize passive heat load on all of the stages. However, instead of the standard 0.085 " diameter coaxial cables, one could use 0.047 " cables or even thinner ones. Using smaller diameter cables is beneficial because the passive heat load scales with the square of the diameter, whereas the attenuation in the cable scales approximately linearly. For the proposed 0.047" SS-SS cables, the passive heat load would be reduced by more than a factor three. Since large attenuation is desired in the drive lines, the active load due to the execution of single-qubit gates would not be increased if one reduced the attenuation of the installed attenuators accordingly. However, the dominant active load at MXC due to flux biasing of the qubits would be increased more than three-fold because the DC resistance of the cables scales inversely with their diameter. The active load in the flux lines could be brought to close to zero though when using superconducting NbTi cables.

As an outlook, we briefly discuss an optimistic scenario in which magnetic flux offsets are brought close to zero by careful magnetic shielding of the quantum processor. Thus avoiding the active load due to DC flux biasing and considering the use of 0.047" diameter cables, the dominant relative load on the CP stage amounts to about $14 \%$, allowing for the operation of about $50 / 0.13 \approx 360$ qubits.

Ultimately, we propose the following modifications to allow for the operation of about one thousand qubits. First, we use superconducting NbTi cables for the flux lines eliminating active load in these lines. Second, we tolerate a CP temperature of about $200 \mathrm{mK}$ at which a cooling power of at least $400 \mu \mathrm{W}$ is available. To maintain the noise photon number below $10^{-3}$ we shift about $7 \mathrm{~dB}$ of attenuation from the $\mathrm{CP}$ to the $\mathrm{MXC}$ stage, increasing the active load on MXC by a factor six. Third, we tolerate a base plate temperature of $30 \mathrm{mK}$ at which the cooling power at MXC is at least doubled to about $40 \mu \mathrm{W}$, while not affecting the noise photon number. We note that in this scenario active loads in the drive lines are the dominating heat load at CP and MXC.

We conclude with the remark that future large-scale dilution refrigerators will likely have several dilution units and/or larger ones to provide sufficient cooling power at the $\mathrm{CP}$ and MXC stages.

\section{Appendix 1: Thermal conductivity of relevant materials and cables}

For the estimation of passive heat loads we used existing data on the thermal conductivity of materials employed in typical cryogenic cables. These thermal conductivities are shown as a function of temperature in Fig. 11. Data for most materials is only available down to $4 \mathrm{~K}$. From $4 \mathrm{~K}$ to $0 \mathrm{~K}$ we use a linear extrapolation to zero. This extrapolation is justified for metals at low temperatures, where the electronic contribution to the thermal conductivity dominates [33]. Although the thermal conductivities of crystallized thermal insulators such as PTFE, and also of superconductors, is expected to have a $T^{3}$ dependence at low temperature, we use a linear interpolation to zero as an upper bound for our estimates.

\section{Appendix 2: Attenuation of different types of coaxial cables}

In addition to the discrete attenuation provided by attenuators, the distributed attenuation of the RF cables contributes to the total attenuation in a given RF line and therefore affects thermal noise photon numbers and active loads. The attenuation in a UT-085-SS-SS cable 


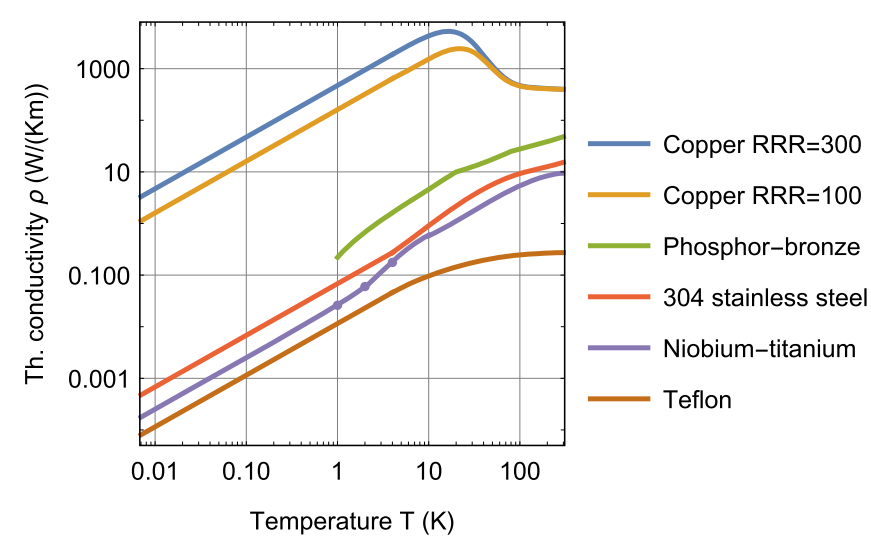

Figure 11 Thermal conductivity of indicated materials as a function of temperature $[54,55]$ on a logarithmic scale. Data for $\mathrm{NbTi}$ in the high temperature regime $(2 \mathrm{~K}<T<300 \mathrm{~K})$ is taken from [56]. The low temperature curve of $\mathrm{NbTi}(T<2 \mathrm{~K})$ is created from three data points [57] and a linear extrapolation towards zero, see text

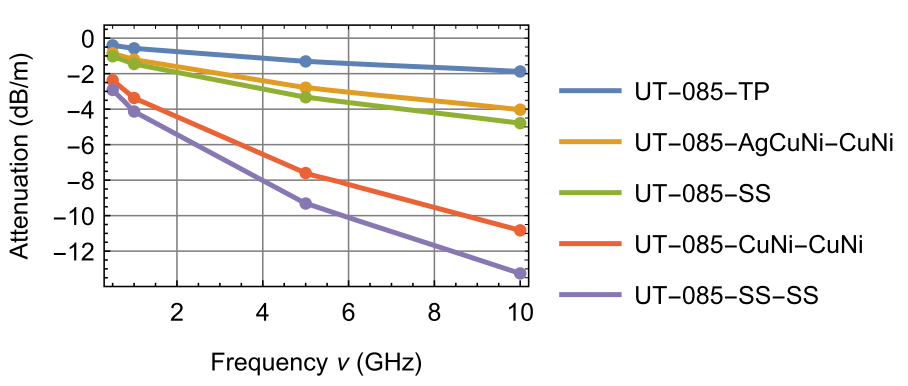

Figure 12 Frequency dependent attenuation of coaxial cables at room temperature for indicated types as specified by the manufacturer $[37,58]$. Solid lines represent linear interpolations between data points

is shown as a function of frequency in Fig. 12 as a purple line. The attenuation of other frequently used cable types is shown for comparison. At low temperature the attenuation is in general slightly reduced, e.g. the attenuation constant of a UT-085-SS-SS cable at $6 \mathrm{GHz}$ reduces from $9.7 \mathrm{~dB} / \mathrm{m}$ at room temperature to $8.7 \mathrm{~dB} / \mathrm{m}$ at $77 \mathrm{~K}$ and $8.2 \mathrm{~dB} / \mathrm{m}$ at $4 \mathrm{~K}$ [36]. For the calculation of the thermal noise photon number and the active loads presented in Fig. 5 and in Fig. 8 we took this temperature dependence into account.

\section{Appendix 3: Scattering parameters of RF lines}

We electrically characterized the installed drive and flux lines by measuring their scattering parameters as a function of frequency using a vector network analyzer (VNA), see Fig. 13. We measured each line from the port on the vacuum flange (Port 1 ) to the port at the MXC flange (Port 2).

The drive lines typically achieve reflection parameters below $-20 \mathrm{~dB}$ from 0 to $15 \mathrm{GHz}$, and below $-25 \mathrm{~dB}$ in the range of 4-8 GHz (Fig. 13(a) and (b)), which is most relevant for the applied microwave pulses. For drive lines including an IR filter (Fig. 13(b)) we choose to position the Eccosorb filter above the attenuator at MXC, see Fig. 3(b). This ensures that the line is well impedance matched also at Port 2 even if the Eccosorb filter itself is not perfectly impedance matched. The transmission coefficients of the drive lines con- 
Figure 13 Typical S-parameters of drive and flux lines. (a) Example of a drive line with a total of $60 \mathrm{~dB}$ attenuation. (b) Example of a drive line with a total of $60 \mathrm{~dB}$ attenuation and an Eccosorb filter having an attenuation of $\sim 5 \mathrm{~dB}$ at $5 \mathrm{GHz}$. (c) Example of a flux line with $10 \mathrm{~dB}$ attenuation, a low pass filter (Minicircuits VLFX1350), and an Eccosorb filter with an attenuation of $\sim 3 \mathrm{~dB}$ at $1 \mathrm{GHz}(\sim 20 \mathrm{~dB}$ at $5 \mathrm{GHz})$

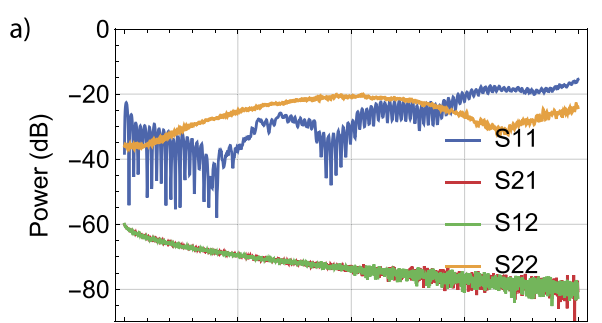

b)

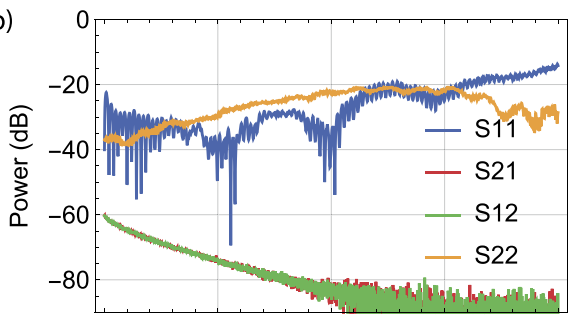

c)

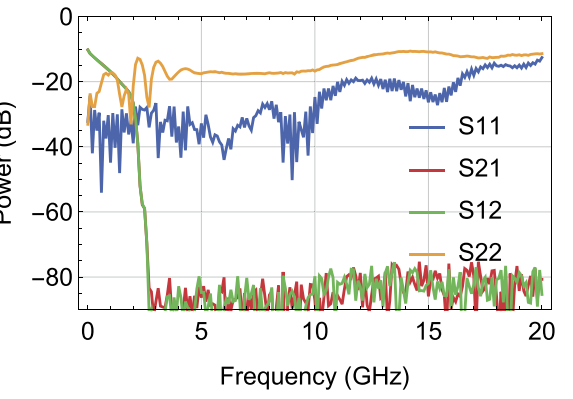

firm that the signal loss at low frequencies is set by the installed attenuators $(3 \times 20 \mathrm{~dB})$. For higher frequencies, the transmission coefficients decrease as expected due to the frequency dependent signal loss in the cables and the Eccosorb filter.

The transmission spectrum of the flux line has its base line at $-10 \mathrm{~dB}$, due to the $10 \mathrm{~dB}$ attenuator installed at the $4 \mathrm{~K}$ stage, and sharply drops at $2 \mathrm{GHz}$ due to a low pass filter with a $3 \mathrm{~dB}$ cutoff frequency of $2.0 \mathrm{GHz}$, see Fig. 13(c). The continuous drop between 0 and $2 \mathrm{GHz}$ is due to a combination of frequency dependent loss in cable and Eccosorb filter. The reflection S22 from the sample side towards the Eccosorb filter is below $-20 \mathrm{~dB}$ in the range of $0-500 \mathrm{MHz}$. Since this range is larger than the typical bandwidth of a flux pulse, possible multiple reflections of flux pulses between the quantum processor and the Eccosorb filter are significantly suppressed. To suppress standing waves between the reflective low pass filter and the quantum processor the absorptive Eccosorb filter is positioned after the low pass filter, see Fig. 3(b).

\section{Appendix 4: Temperature sensors and heaters}

We use a Platinum PT100 temperature sensor at $50 \mathrm{~K}$, Cernox CX-1010 sensors at $4 \mathrm{~K}$ and Still, and RuO2 RX-102B sensors at CP and MXC (all sensors are from Lake Shore Cryotronics). All except the sensor at CP were pre-installed by the manufacturer. The sensors are read out using the temperature controller model 372 from Lake Shore Cryotronics. We also installed resistive heaters on the $50 \mathrm{~K}$ and the CP stages that had none installed by the manufacturer. On the $50 \mathrm{~K}$ stage we installed a $25 \Omega, 100 \mathrm{~W}$ heater (HTR-25-100, Lake Shore Cryotronics), whereas on the CP we installed a $50 \Omega, 50 \mathrm{~W}$ heater (HTR-50, 
Figure 14 Performance of the two pulse tube cooler system in the DR. The temperature responses on the $4 \mathrm{~K}$ and $50 \mathrm{~K}$ stages are plotted for each of the nine combinations of applied power $\left(P_{4 \mathrm{~K}}, P_{50} \mathrm{~K}\right)$

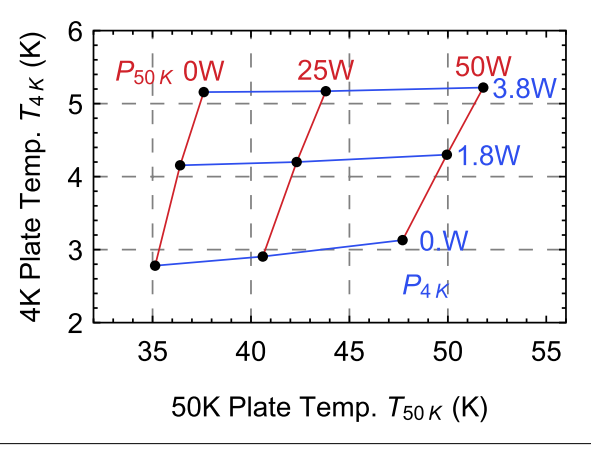

Lake Shore Cryotronics). For mounting, the cartridge heaters were clamped in a circular hole in a rectangular $\mathrm{Cu}$ piece which was then mounted to the corresponding plate in the cryostat.

\section{Appendix 5: Cooling power of pulse tube coolers}

We measured the cooling power of the pulse tube coolers installed in the dilution refrigerator system by the manufacturer at the $50 \mathrm{~K}$ and $4 \mathrm{~K}$ stages in the regime of large relative temperatures using the method described in Sect. 4.1. Since the cooling power of the pulse tube coolers (2x PT420 from Cryomech) at each stage also depends on the temperature of the respective other stage [59], we measure the temperature increases in a twodimensional parameter space spanned by the powers applied to the $50 \mathrm{~K}$ and $4 \mathrm{~K}$ stages, see Fig. 14. The data has been taken without circulating mixture for safety reasons. The obtained data qualitatively agrees with the specifications of the manufacturer Cryomech [59]. We note that the extracted cooling powers of $1.5 \mathrm{~W}$ at a temperature of $4.2 \mathrm{~K}$ of the $4 \mathrm{~K}$ stage and of $30 \mathrm{~W}$ at a temperature of $45 \mathrm{~K}$ of the $50 \mathrm{~K}$ stage are lower than the values of $3.6 \mathrm{~W}$ and $100 \mathrm{~W}$ specified by the manufacturer, ${ }^{\mathrm{g}}$ respectively. The main reason for the discrepancy at the $4 \mathrm{~K}$ stage is that we measure the temperature at the $4 \mathrm{~K}$ plate, while the manufacturer specifies the temperature at the cold head of the pulse tube cooler. It is plausible that a temperature difference of the order of one Kelvin arises between plate and cold head when considering that the thermal contact resistance at these temperatures is of the order of a K/W. The main reason for the discrepancy at the $50 \mathrm{~K}$ stage is the radiative load from the room temperature vacuum can on the $50 \mathrm{~K}$ shield, see Sect. 2.3. In addition, heat conduction through the structural support of the dilution refrigerator contributes to a reduced cooling power on both stages.

\section{Appendix 6: Offset passive loads}

To verify that an increased temperature at stage $i-1$ merely creates an offset passive load on stage $i$ without altering the functional dependence between cooling power and temperature on stage $i$, we measured $T_{i}$ vs. $P_{i}$ curves for $P_{i-1} \neq 0$. An exception is the Still stage since its temperature regulates the flow rate of the ${ }^{3} \mathrm{He}$ and thus the cooling power at the $\mathrm{CP}$ and MXC stages. The resulting data sets are offset along the applied power axis compared to their $P_{i-1}=0$ counterparts of Fig. 7, see Fig. 15. Beyond the offset, the functional form of the $P_{i-1} \neq 0$ data is virtually indistinguishable from the $P_{i-1}=0$ data, demonstrating that an increased temperature on stage $i-1$ merely creates an offset passive load on 
Figure 15 Measurement of offset passive loads. Temperature increases on the (a) $4 \mathrm{~K}$, (b) Still, (c) MXC plates of the DR as a function of applied power to these stages for zero and non-zero applied power to the next higher stage
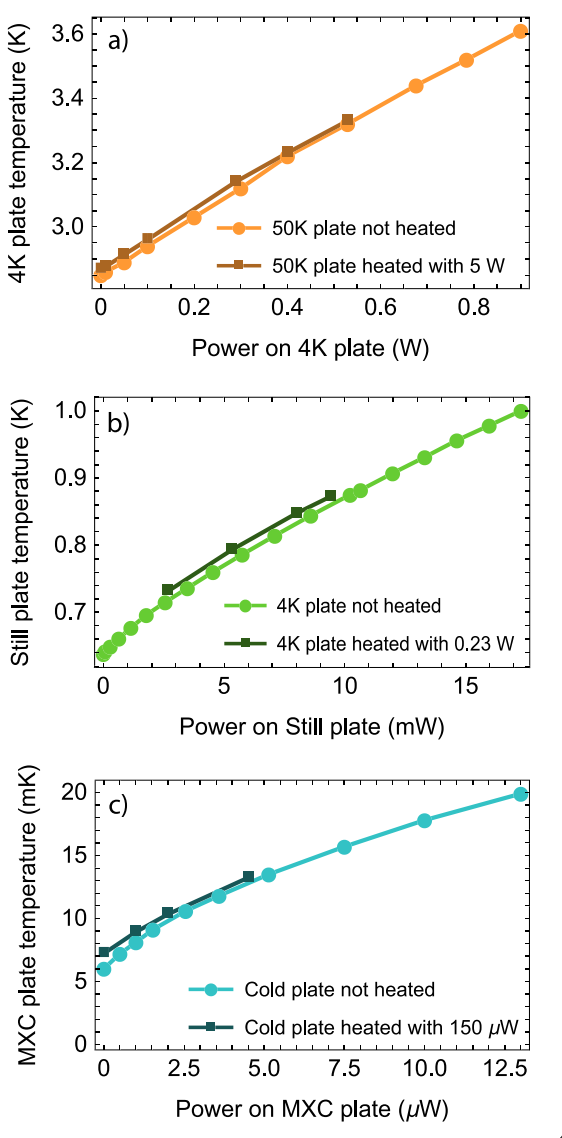

stage $i$. The determined offsets of $(0.02 \mathrm{~W}, 0.6 \mathrm{~mW}, 0.5 \mu \mathrm{W})$, for the $4 \mathrm{~K}$, Still and MXC stages respectively, agree with the offsets $\left(\partial P_{i} / \partial T_{i-1}\right) \Delta T_{i-1}$ calculated from the reference data. We subtracted these offset loads $\left(\partial P_{i} / \partial T_{i-1}\right) \Delta T_{i-1}$ from the observed loads when extracting the passive loads of the cable trees, see discussion in Sect. 4.2. Data for the CP stage was not taken because for the determination of the passive loads the Still temperature was kept at the same value to realize constant flow of ${ }^{3} \mathrm{He}$ and thus constant cooling power.

\section{Acknowledgements}

The authors thank L. DiCarlo, Y. Salathé, G. Norris, C. K. Andersen, and S. Gasparinetti for useful discussions, and M. Frey for support during the installation of the dilution refrigerator.

\section{Funding}

This work is supported by the Office of the Director of National Intelligence (ODNI), Intelligence Advanced Research Projects Activity (IARPA), via the U.S. Army Research Office grant W91 1NF-16-1-0071 and by ETH Zurich. The views and conclusions contained herein are those of the authors and should not be interpreted as necessarily representing the official policies or endorsements, either expressed or implied, of the ODNI, IARPA, or the U.S. Government.

\section{Availability of data and materials}

The datasets used and/or analysed during the current study are available from the corresponding author on reasonable request.

\section{Competing interests}

The authors declare that they have no competing interests.

\section{Authors' contributions}

The design of the cryogenic setup was developed by SK, PK, RK, JL, CE and AW. The setup was built by SK, SS and JH. The experiments were performed by SK and SS. The data was analyzed and interpreted by SK, SS, PK and PM. The manuscript 
was written by SK, SS, CE and AW. The project was led by AW. All authors commented on the manuscript. All authors have read and approved the final manuscript.

\section{Endnotes}

a Silver plated copper weld, also referred to as silver plated copper clad steel per ASTM B-501. It is composed of a steel core and a copper cladding. For our estimate, we model it as a single core Cu wire with an RRR of 100.

b For clarity, NbTi twisted pairs are considered to consist of pure $\mathrm{NbTi}$, although they are typically embedded in a CuNi matrix for mechanical stability.

c Smaller diameter cables of the same type would further reduce the passive heat load, but introduce a larger frequency dependence in the $\$ 21$ transmission parameter.

d When using only a HEMT amplifier as a cryogenic amplifier (and not a TWPA), an ultra-low noise amplifier with a noise temperature of about $100 \mathrm{~K}$ is recommended.

e Choosing a smaller coupling ratio would decrease the transmission of the readout signal through the directional coupler accordingly.

f We assume the dielectric (Teflon) to be thermalized with the center conductor because the thermal expansion coefficient of Teflon is a factor of six larger than the one of stainless steel.

$g$ The quoted cooling powers are for two PT420 pulse tube coolers with remote motor.

\section{Publisher's Note}

Springer Nature remains neutral with regard to jurisdictional claims in published maps and institutional affiliations.

Received: 21 November 2018 Accepted: 4 April 2019 Published online: 28 May 2019

\section{References}

1. Devoret MH, Schoelkopf RJ. Superconducting circuits for quantum information: an outlook. Science. 2013;339:1169-74. http://science.sciencemag.org/content/339/6124/1169.

2. Awschalom DD, Bassett LC, Dzurak AS, Hu EL, Petta JR. Quantum spintronics: engineering and manipulating atom-like spins in semiconductors. Science. 2013;339:1174-9. http://science.sciencemag.org/content/339/6124/1174.

3. Yan F, et al. The flux qubit revisited to enhance coherence and reproducibility. Nat Commun. 2016;7:12964. https://www.nature.com/articles/ncomms12964.

4. Yeh J-H, LeFebvre J, Premaratne S, Wellstood FC, Palmer BS. Microwave attenuators for use with quantum devices below 100 mK. J Appl Phys. 2017;121:224501. https://aip.scitation.org/doi/abs/10.1063/1.4984894.

5. IBM Q experience. https://quantumexperience.ng.bluemix.net/qx/devices. Accessed: 2018-06-15.

6. Otterbach JS, et al. Unsupervised machine learning on a hybrid quantum computer. 2017. arXiv:1712.05771 [quant-ph].

7. Ithier G, et al. Decoherence in a superconducting quantum bit circuit. Phys Rev B. 2005;72:134519. http://link.aps.org/doi/10.1103/PhysRevB.72.134519.

8. Catelani G, et al. Quasiparticle relaxation of superconducting qubits in the presence of flux. Phys Rev Lett. 2011;106:077002. http://link.aps.org/doi/10.1103/PhysRevLett.106.077002.

9. Barends R, et al. Minimizing quasiparticle generation from stray infrared light in superconducting quantum circuits. Appl Phys Lett. 2011:99:113507. http://scitation.aip.org/content/aip/journal/apl/99/11/10.1063/1.3638063.

10. Córcoles AD, et al. Protecting superconducting qubits from radiation. Appl Phys Lett. 2011;99:181906. http://scitation.aip.org/content/aip/journal/apl/99/18/10.1063/1.3658630.

11. Pop IM, et al. Coherent suppression of electromagnetic dissipation due to superconducting quasiparticles. Nature. 2014;508:369-72. http://www.nature.com/nature/journal/v508/n7496/full/nature13017.html.

12. Kreikebaum JM, Dove A, Livingston W, Kim E, Siddiqi I. Optimization of infrared and magnetic shielding of superconducting TiN and Al coplanar microwave resonators. Supercond Sci Technol. 2016;29:104002. http://stacks.iop.org/0953-2048/29/i=10/a=104002.

13. Serniak K, Hays M, de Lange G, Diamond S, Shankar S, Burkhart LD, Frunzio L, Houzet M, Devoret MH. Hot nonequilibrium quasiparticles in transmon qubits. Phys Rev Lett. 2018;121:157701. https://link.aps.org/doi/10.1103/PhysRevLett.121.157701.

14. Lukashenko A, Ustinov AV. Improved powder filters for qubit measurements. Rev Sci Instrum. 2008;79:014701 http://scitation.aip.org/content/aip/journal/rsi/79/1/10.1063/1.2827515.

15. Geerlings KL. Improving coherence of superconducting qubits and resonators. PhD dissertation. Yale University; 2013.

16. Koch J, et al. Charge-insensitive qubit design derived from the Cooper pair box. Phys Rev A. 2007;76:042319. http://link.aps.org/doi/10.1103/PhysRevA.76.042319.

17. Song C, et al. Microwave response of vortices in superconducting thin films of Re and Al. Phys Rev B. 2009;79:174512 https://link.aps.org/doi/10.1103/PhysRevB.79.174512.

18. Nsanzineza I, Plourde B. Trapping a single vortex and reducing quasiparticles in a superconducting resonator. Phys Rev Lett. 2014;113:117002. https://link.aps.org/doi/10.1103/PhysRevLett.113.117002.

19. Hutchings $M$, et al. Tunable superconducting qubits with flux-independent coherence. Phys Rev Appl. 2017:8:044003. https://link.aps.org/doi/10.1103/PhysRevApplied.8.044003.

20. Blais A, Huang R-S, Wallraff A, Girvin SM, Schoelkopf RJ. Cavity quantum electrodynamics for superconducting electrical circuits: an architecture for quantum computation. Phys Rev A. 2004;69:062320. http://link.aps.org/doi/10.1103/PhysRevA.69.062320

21. DiCarlo $L$, et al. Demonstration of two-qubit algorithms with a superconducting quantum processor. Nature 2009;460:240-4. https://www.nature.com/articles/nature08121.

22. Barends $R$, et al. Superconducting quantum circuits at the surface code threshold for fault tolerance. Nature. 2014;508:500-3. http://www.nature.com/nature/journal/v508/n7497/full/nature13171.html. 
23. Caldwell SA, et al. Parametrically activated entangling gates using transmon qubits. Phys Rev Appl. 2018;10:034050. https://link.aps.org/doi/10.1103/PhysRevApplied.10.034050.

24. Chen Y, et al. Qubit architecture with high coherence and fast tunable coupling. Phys Rev Lett. 2014;113:220502. https://link.aps.org/doi/10.1103/PhysRevLett.113.220502.

25. McKay DC, et al. Universal gate for fixed-frequency qubits via a tunable bus. Phys Rev Appl. 2016;6:064007. https://link.aps.org/doi/10.1103/PhysRevApplied.6.064007.

26. Wallraff A, et al. Approaching unit visibility for control of a superconducting qubit with dispersive readout. Phys Rev Lett. 2005;95:060501. https://link.aps.org/doi/10.1103/PhysRevLett.95.060501.

27. Vijay R, Slichter DH, Siddiqi I. Observation of quantum jumps in a superconducting artificial atom. Phys Rev Lett. 2011;106:110502. https://link.aps.org/doi/10.1103/PhysRevLett.106.110502.

28. Walter T, et al. Rapid high-fidelity single-shot dispersive readout of superconducting qubits. Phys Rev Appl. 2017;7:054020. https://link.aps.org/doi/10.1103/PhysRevApplied.7.054020.

29. Leiden cryogenics. http://www.leiden-cryogenics.com/. Accessed: 2018-06-07.

30. Oxford instruments. https://nanoscience.oxinst.com/. Accessed: 2018-06-07.

31. Bluefors cryogenics. http://www.bluefors.com/. Accessed: 2018-06-07.

32. Parma, Design VC. 2015. arXiv:1501.07154 [physics].

33. Duthil P. Material properties at low temperature. 2015. arXiv:1501.07100 [cond-mat, physics:physics].

34. Uhlig K. $3 \mathrm{He} / 4 \mathrm{He}$ dilution refrigerator with pulse-tube refrigerator precooling. Cryogenics. 2002;42:73-7. http://www.sciencedirect.com/science/article/pii/S0011227502000024.

35. Uhlig K. Dry dilution refrigerator with high cooling power. AIP Conf Proc. 2008;985:1287-91. https://aip.scitation.org/doi/abs/10.1063/1.2908485.

36. Kurpiers P, Walter T, Magnard P, Salathe Y, Wallraff A. Characterizing the attenuation of coaxial and rectangular microwave-frequency waveguides at cryogenic temperatures. EPJ Quantum Technol. 2017;4:8. https://epjquantumtechnology.springeropen.com/articles/10.1140/epjqt/s40507-017-0059-7.

37. COAX Co. Ltd. http://www.coax.co.jp/en/cryogenic. Accessed 2018-08-19.

38. Gardiner C, Zoller P. Quantum noise: a handbook of Markovian and non-Markovian quantum stochastic methods with applications to quantum optics. 3rd ed. Springer series in synergetics. Berlin Heidelberg: Springer; 2004. www.springer.com/de/book/9783540223016.

39. Pechal M. Microwave photonics in superconducting circuits. Ph.D. thesis. Zurich: ETH; 2016. https://www.research-collection.ethz.ch/handle/20.500.11850/155858.

40. Chen Z, et al. Measuring and suppressing quantum state leakage in a superconducting qubit. Phys Rev Lett. 2016;116:020501. https://link.aps.org/doi/10.1103/PhysRevLett.116.020501.

41. Carmichael H. Statistical methods in quantum optics 1: master equations and Fokker-Planck equations. Physics and Astronomy Online Library.

42. Martinis JM, Geller MR. Fast adiabatic qubit gates using only $\sigma_{z}$ control. Phys Rev A. 2014;90:022307. http://link.aps.org/doi/10.1103/PhysRevA.90.022307.

43. BlueFors Cryogenics Oy Ltd. Bluefors LD400 Cooling power. 2014

44. Salerno LJK. Thermal contact conductance. 1997. Tech. Rep. https://ntrs.nasa.gov/search.jsp?R=19970026086.

45. Kumar $P$, et al. Origin and reduction of $\$ 1 / \$ \$$ magnetic flux noise in superconducting devices. Phys Rev Appl. 2016;6:041001. https://link.aps.org/doi/10.1103/PhysRevApplied.6.041001.

46. de Graaf $\mathrm{S}$, et al. Direct identification of dilute surface spins on $\mathrm{Al}_{2} \mathrm{O}_{3}$ : origin of flux noise in quantum circuits. Phys Rev Lett. 2017;118:057703. https://link.aps.org/doi/10.1103/PhysRevLett.118.057703.

47. Sendelbach S, Hover D, Mück M, McDermott R. Complex inductance, excess noise, and surface magnetism in dc SQUIDs. Phys Rev Lett. 2009;103:117001. https://link.aps.org/doi/10.1103/PhysRevLett.103.117001.

48. Eichler $C$, Bozyigit $D$, Wallraff $A$. Characterizing quantum microwave radiation and its entanglement with superconducting qubits using linear detectors. Phys Rev A. 2012;86:032106. https://ink.aps.org/doi/10.1103/PhysRevA.86.032106.

49. Macklin C, et al. A near-quantum-limited Josephson traveling-wave parametric amplifier. Science. 2015;350:307-10 http://science.sciencemag.org/content/350/6258/307.

50. Heinsoo J, et al. Rapid high-fidelity multiplexed readout of superconducting qubits. Phys Rev Appl. 2018;10:034040. https://link.aps.org/doi/10.1103/PhysRevApplied.10.034040.

51. Betts DS. An introduction to Millikelvin technology. Cambridge studies in low temperature physics. Cambridge: Cambridge University Press; 1989

52. BlueFors Cryogenics, BF-XLD-Series User manual. 2016

53. Schwark M, et al. Ortho-para conversion of hydrogen in copper as origin of time-dependent heat leaks. J Low Temp Phys. 1983;53:685-94. https://doi.org/10.1007/BF00684000.

54. NIST cryogenic material properties database. http://cryogenics.nist.gov/Papers/Cryo_Materials.pdf. Accessed: 2016-05-06.

55. Lake Shore Cryotronics. https://www.lakeshore.com/products/cryogenic-accessories/wire/pages/Specifications.aspx. Accessed: 2018-02-16.

56. Flachbart K, Feher A, Janos S, Malek Z, Ryska A. Thermal conductivity of NbTi alloy in the low-temperature range. Phys Status Solidi (b). 1978:85:545-51.

57. Duthil P. Materials properties at low temperature. https://cas.web.cern.ch/schools/erice-2013 (2013). Accessed: 2018-10-04.

58. Carlisle interconnect technologies.

https://www.carlisleit.com/CITSite/media/CITMedia/Documentation/Semi-Rigid_Catalog.pdf. Accessed: 2018-10-04.

59. Cryomech Inc. 2018. http://www.cryomech.com/cryorefrigerators/pulse-tube/pt420/. Accessed: 2018-08-22. 